\title{
Multivariable Nonlinear Control of a Continuous Polymerization Reactor: an Experimental Study
}

\author{
Masoud Soroush and Costas Kravaris \\ Dept. of Chemical Engineering, The University of Michigan, Ann Arbor, MI 48109
}

\begin{abstract}
This experimental work concerns the multivariable nonlinear control of a continuous stirred-tank polymerization reactor. The globally linearizing control (GLC) method is implemented to control conversion and temperature in the reactor in which the solution polymerization of methyl methacrylate takes place. Control of conversion and temperature is achieved by manipulating the flow rate of an inlet initiator stream and two coordinated heat input variables. Conversion is inferred from on-line measurements of density and temperature. A reduced-order state observer is utilized to estimate the concentrations of monomer, initiator and solvent in the reactor. The concentration estimates are used in the control law. This study demonstrates the considerable computational efficiency of the nonlinear controller, which is implemented on a microcomputer. The experimental results show the excellent performance of the controller in the presence of active state and input constraints. A systematic approach is also given for the synthesis of output feedback controllers within the GLC framework for processes with secondary outputs.
\end{abstract}

\section{Introduction}

The macromolecular structure and the material properties of polymers are determined mainly at the synthesis stage. Therefore, there is a great incentive for the efficient operation of polymerization reactors. The crucial role that good control can play in achieving an efficient operation is recognized well in the polymerization literature (Amrehn, 1977; Elicabe and Meira, 1988; MacGregor, 1986; Ray, 1986, 1992; Tirrell et al., 1987).

Control of polymerization reactors has always been a challenging task mostly because of (a) the inadequacy of on-line sensors with fast sampling rate and small time delay, and (b) the complex nonlinear and strongly interactive behavior of polymerization reactors.

The lack of reliable and easily-available on-line measurements, from which polymer properties can be inferred, has motivated a considerable research effort in the three major directions:

- The development of new on-line sensors [lists of currently-

Current address of M. Soroush: Dept. of Chemical Engineering, Drexe! University, Philadelphia, PA 19104. available on-line sensors are provided by Chien and Penlidis (1990) and Ray (1992)].

- The development of state estimation techniques capable of estimating unmeasurable polymer properties (see Elicabe and Meira, 1988; Ray, 1992, and the references therein). A list of measurements from which certain polymer properties can be observed and/or detected is given by Ray (1986).

- The study and understanding of the qualitative and/or quantitative relations between easily-available on-line measurements like density, viscosity and refractive index, and certain polymer properties like conversion and average molecular weights (Ponnuswamy et al., 1986; Schork and Ray, 1983).

Despite the significant progress in these areas, the inadequacy of currently-available on-line sensors is still a major barrier to efficient control of polymerization reactors.

Polymerization reactors are known as highly nonlinear processes, and the need for nonlinear control has been recognized in the polymerization literature (Elicabe and Meira, 1988; MacGregor, 1986; Ray, 1986). Polymerization reactor models have been used extensively to test the performance of a variety of control techniques through simulations. However, only a very limited number of experimental closed-loop control stud- 
ies have been reported in the literature. These experimental studies have been limited mainly to temperature and pressure control because of the difficulty involved in getting on-line measurements in the viscous polymerizing mixtures. In addition to the GLC (Soroush and Kravaris, 1992a), adaptive, model-predictive and other conventional controllers have been tested experimentally, primarily in batch polymerization (Bejger et al., 1981; Inglis et al., 1991; Ponnuswamy et al., 1987; Tirrell and Gromley, 1981; Tzouanas and Shah, 1989). Very recently, application of the nonlinear geometric control method to industrial polymerization reactors has also been reported (McAuley and MacGregor, 1992; Singstad et al., 1992).

In this study, the GLC method (Kravaris and Soroush, 1990) is implemented experimentally to control temperature and conversion in a continuous stirred-tank polymerization reactor. The solution polymerization of methyl methacrylate (MMA) in toluene is initiated by azo-bis-isobutyronitrile (AIBN). The control of temperature and conversion is achieved by manipulating a net heat input to the jacket and the flow rate of an inlet initiator stream. Conversion is inferred from on-line measurements of density and the temperature at which density is measured. Some preliminary results of this experimental study were presented in (Soroush and Kravaris, 1992b). This work is the first experimental study in which a MIMO chemical reactor is controlled by a geometric control method.

This article starts with the description of the experimental system, and then a correlation for inferring conversion from density and temperature measurements is proposed. After a brief review of multi-input/multi-output (MIMO) GLC and development of a mathematical model of the process, the controller is synthesized. Finally, the issues involved in the realtime implementation of the control law are discussed, as well as the performance of the controller.

\section{Experimental System}

Figure 1 shows the experimental system. The reactor is a 3$\mathrm{L}$ jacketed glass vessel, and the computer is a DTK TECH1000. The data acquisition software is ASYST (version 3.10) from Asyst Software Technologies, Inc. The reactor temperature is measured by a resistance temperature detector (RTD). The heating/cooling system of the reactor consists of an electrical heater, circulating tubes, a pneumatic control valve, a flowmeter, a circulating pump, and two RTDs in the jacket side. This experimental system includes all the components of our batch experimental system (Soroush and Kravaris, 1992a); therefore, only the extra equipment of this CSTR system will be described here.

Additionally, the CSTR experimental system is equipped with a Micro Motion Coriolis Metering System, which can measure on-line: (i) the density of the reacting mixture ( $\rho$ ); (ii) the temperature at which the density is measured $\left(T_{d}\right)$; and (iii) the flow rate of the stream passing through the sensor. In this study, only the first two measurements are used for control purposes. The densitometer system consists of a DS012S Mass Flow and Density Sensor, an RFT9712 Smart Family Transmitter, and a DMS Density Monitoring System, all from Micro Motion, Inc. The Density Sensor includes vibrating U-shaped tubes. The density is calculated on the basis of the resonant frequency of the vibrating tubes and the fluid in the tubes. The Monitoring System provides the density and temperature

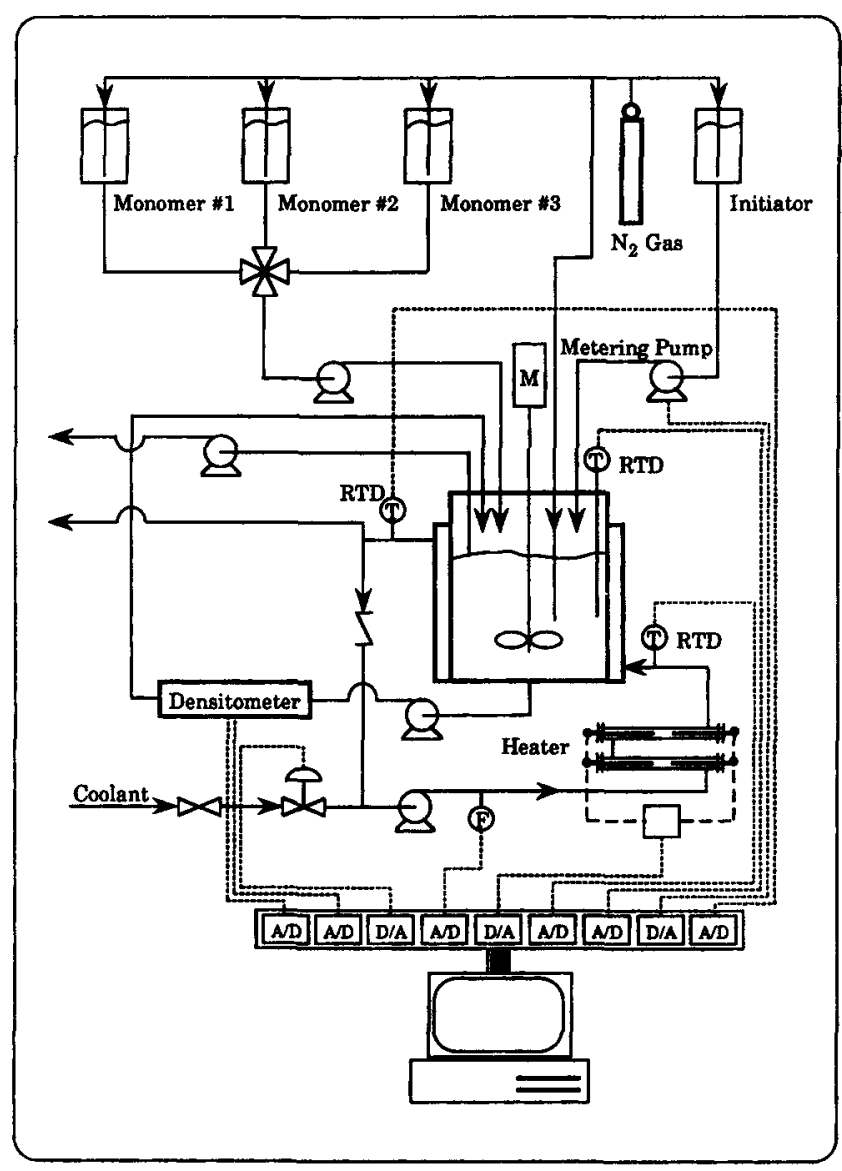

Figure 1. Experimental system.

measurements as 4-20-mA analog signals and shows the actual density and temperature measurements in user-selectable units. On the basis of the specifications reported by the manufacturer, the accuracy of the density and temperature measurements are as follows: $\pm 2 \mathrm{~kg} \cdot \mathrm{m}^{-3}$ and $\pm 1.4 \mathrm{~K}$. When there is a sharp change in the temperature of the stream passing through the densitometer sensor (such as during the start-up period), during the transient period, the error in the density measurements exceeds the error limit reported by the manufacturer (sensor gives overmeasured signals). The density measurements become reliable when the sensor gets thermally stabilized.

As Figure 1 shows, to measure the density of the reacting mixture, the fluid is pumped out from the bottom of the reactor at a relatively high flow rate $\left(\approx 1.7 \times 10^{-5} \mathrm{~m}^{3} \cdot \mathrm{s}^{-1}\right)$, goes through the sensor and is totally recycled to the reactor. For the deadtime in the density measurement to be less than the sampling period of $5 \mathrm{~s}$, the flow rate of the sensor stream is chosen to be sufficiently high without deteriorating the quality of density measurement. Note that at nominal operating conditions, there is almost $10 \mathrm{~K}$ difference between the reactor temperature and the temperature measured by the densitometer; this suggests relatively significant heat losses from the sensor and the circulating tube to the environment. This heat loss will not be accounted for in the modeling. The controller is robust to this modeling error. Two identical gear pumps (model 415120 from Miropump Corp.), which work in parallel, are employed to pump the reacting mixture through the densitometer sensor. Because of the significant pressure drop across the sensor, after 
Table 1. Other Parameters of the Experimental System

$$
\begin{aligned}
T_{\text {lms }} & =2.952 \times 10^{2} \mathrm{~K} \\
F_{i_{\max }} & =2.80 \times 10^{-8} \mathrm{~m}^{3} \cdot \mathrm{s}^{-1} \\
V & =1.2 \times 10^{-3} \mathrm{~m}^{3} \\
F_{m} & =2.70 \times 10^{-7} \mathrm{~m}^{3} \cdot \mathrm{s}^{-1} \\
T_{\infty} & =2.952 \times 10^{2} \mathrm{~K}
\end{aligned}
$$

1,600 -s operation, the thermal switch of the pump gets activated and the pump stops. Therefore, during the operation, while one of these pumps operates, the other one is on standby.

In this experimental system, there are two outlet and two inlet streams. One of the outlet streams, which goes through the densitometer (densitometer stream), is totally recycled. The second outlet (CSTR product) stream is pumped out from top of the reactor through a glass tube, which is extended up to the level corresponding to the desired CSTR volume of $1.2 \times 10^{-3} \mathrm{~m}^{3}$. The product stream is pumped out by a metering pump [model RHVRH0CTC, from Fluid Metering, Inc. (FMI)] at a desirable constant flow rate $\left(3.0 \times 10^{-7} \mathrm{~m}^{3} \cdot \mathrm{s}^{-1}\right)$ and is collected in a drum. One of the inlet streams is the monomer stream, which is fed to the reactor by a metering pump (the same type as the product pump) at a desirable constant flow rate $\left(2.7 \times 10^{-7} \mathrm{~m}^{3} \cdot \mathrm{s}^{-1}\right)$. The second inlet stream is the initiator stream. In this experiment, in addition to the net heat input to the jacket by the heater and inlet cooling water, the inlet initiator flow rate $\left(F_{i}\right)$ is also manipulated; it is adjusted by a metering pump (model QV650RH0CTCLF, from FMI) in proportion to the 4-20-mA signal coming from a $D / A$ port. Since (a) the flow rate of the product stream is slightly higher than the maximum sum of the flow rates of the two inlet streams, (b) the product stream is pumped out through a tube that is extended up to the level corresponding to the desired volume, and (c) the product pump can handle the presence of bubbles in the product stream, the liquid level remains constant throughout the operation.

The monomer and initiator solution streams are supplied by feed bottles. Prior to and during the operation, oxygen (a reaction inhibitor) is purged from the fluids inside the bottles by bubbling nitrogen through and agitating the solutions using magnetic stirrers. Other parameters of the experimental system are given in Table 1.

\section{Dynamics of the Control Elements}

The dynamics of the control elements (control valve, RTDs, control valve pressure transducer, densitometer, initiator stream metering pump, and heater) are very fast compared to the dynamics of the reactor and the jacket. Therefore, these dynamics will be neglected in the model development of the system. A study of the steady-state input/output behavior of these elements showed that the heater (signal/power), the densitometer (density/signal), the product metering pump (signal/ flow), the RTDs (temperature/signal), and the pressure transducer (signal/air pressure) are linear. Therefore, the signals from or to these elements are calibrated by linear equations. However, the steady-state behavior of the air-to-close control valve (air pressure/flow) is nonlinear and can be represented by a quadratic equation. This nonlinearity is accounted for in the controller system using the quadratic calibration equation for calculating the actual flow rate from the corresponding digital signal and vice versa.

\section{Density-Temperature-Conversion Correlation}

In this study, one of the controlled outputs is the conversion $x_{p}$, which is defined as the solvent-free mass fraction of polymer in the reactor. Therefore, to apply the feedback controller to the polymerization reactor, we need the on-line measurement of conversion. The conversion $\left(x_{p}\right)$ can be inferred from the density and temperature measurements. There are many approaches (Ponnuswamy et al., 1986; Schork and Ray, 1983; Tzouanas and Shah, 1989) that can be used to infer conversion during the course of polymerization. In this study, we use an approach which is based on a total mass balance for the CSTR (like the one given in Ponnuswamy et al., 1986). The proposed approach involves the use of the relation:

$$
x_{p}=\frac{1}{\epsilon}\left(\frac{\rho_{m s}}{\rho}-1\right)
$$

which is indeed a simple overall mass balance for the reactor at steady-state conditions. Here, $\epsilon$ is the volume expansion factor given by:

$$
\epsilon=\phi_{m s s}\left(\frac{\rho_{m}}{\rho_{p}}-1\right)
$$

and $\rho_{m s}$ is the density of inlet monomer stream given by:

$$
\rho_{m s}=\phi_{m_{m s}} \rho_{m}+\left(1-\phi_{m_{m s}}\right) \rho_{s}
$$

where (Louie et al., 1985)

$$
\begin{aligned}
& \rho_{m}=968-1.225\left(T_{d}-273.2\right) \\
& \rho_{s}=883-0.900\left(T_{d}-273.2\right)
\end{aligned}
$$

The above approach is used to infer conversion from density and temperature measurements.

\section{Mathematical Model}

The GLC is a model-based control method; therefore, we need a mathematical description of the process to synthesize the nonlinear controller. The following mathematical model is developed based on mass and energy balances.

\section{Reactor dynamics}

For free-radical polymerization of MMA initiated by AIBN in toluene the reaction rate expressions are the same as in (Soroush and Kravaris, 1992a):

$$
\begin{aligned}
R_{m} & =-C_{m} \xi_{0}\left(k_{P}+k_{f_{m}}\right), \\
R_{i} & =-k_{i} C_{i}, \\
R_{H} & =\left(-\Delta H_{P}\right) k_{P} \xi_{0} C_{m},
\end{aligned}
$$


where

$$
\xi_{0}=\left(\frac{2 f k_{i} C_{i}}{k_{t}}\right)^{0.5}
$$

In this study, to account for gel and glass effects, the same gel and glass effect models used by Schmidt and Ray (1981) are employed. Therefore, the overall propagation and termination rate constants are given by:

$$
k_{t}=g_{t} k_{t_{0}}, \quad k_{P}=g_{P} k_{P_{0}},
$$

where the factors $g_{r}$ and $g_{P}$ are given by:

$g_{t}= \begin{cases}\alpha_{t_{11}} \exp \left[\alpha_{t_{12}} V_{f}-\alpha_{t_{13}}(T-273.2)\right], & V_{f}>\alpha_{v_{1}}-\alpha_{v_{2}}(T-273.2) \\ \alpha_{t_{21}} \exp \left(\alpha_{t_{22}} V_{f}\right) & V_{f} \leq \alpha_{v_{1}}-\alpha_{v_{2}}(T-273.2)\end{cases}$

$g_{P}= \begin{cases}1, & V_{f}>0.05 \\ \alpha_{p_{21}} \exp \left(\alpha_{p_{22}} V_{f}\right), & V_{f} \leq 0.05\end{cases}$

where $\alpha_{i}$ 's are constant parameters. The total free volume, $V_{f}$, is defined by the contributions of monomer, polymer and solvent as follows:

$$
V_{f}=V_{f_{p}} \phi_{p}+V_{f_{m}} \phi_{m}+V_{f_{s}} \phi_{s}
$$

$\phi_{p}, \phi_{m}$, and $\phi_{s}$ are the volume fractions of polymer, monomer and solvent in the mixture defined by:

$$
\begin{aligned}
& \phi_{p}=\frac{\frac{\mu_{1}}{\rho_{p}}}{\frac{\mu_{1}}{\rho_{p}}+\frac{C_{m} M_{m}}{\bar{\rho}_{m}}+\frac{C_{s} M_{s}}{\bar{\rho}_{s}}}, \\
& \phi_{m}=\frac{\frac{C_{m} M_{m}}{\bar{\rho}_{m}}}{\frac{\mu_{1}}{\rho_{p}}+\frac{C_{m} M_{m}}{\bar{\rho}_{m}}+\frac{C_{s} M_{s}}{\bar{\rho}_{s}}}, \\
& \phi_{s}=\frac{\frac{C_{s} M_{s}}{\bar{\rho}_{s}}}{\frac{\mu_{1}}{\rho_{p}}+\frac{C_{m} M_{m}}{\bar{\rho}_{m}}+\frac{C_{s} M_{s}}{\bar{\rho}_{s}},}
\end{aligned}
$$

and

$$
\begin{aligned}
& V_{f_{p}}=0.025+\alpha_{p}\left(T-T_{g p}\right), \\
& V_{f_{m}}=0.025+\alpha_{m}\left(T-T_{g m}\right), \\
& V_{f_{s}}=0.025+\alpha_{s}\left(T-T_{g s}\right)
\end{aligned}
$$

Table 2. Gel and Glass Effect Model Parameters (Schmidt and Ray, 1981)

$\begin{aligned} \alpha_{t_{1}} & =1.0575 \times 10^{-1} & \alpha_{t_{12}} & =1.7150 \times 10^{1} \\ \alpha_{t_{13}} & =1.7150 \times 10^{-2} & \alpha_{t_{11}} & =2.3000 \times 10^{-6} \\ \alpha_{t_{22}} & =7.5000 \times 10^{1} & \alpha_{p_{21}} & =7.1000 \times 10^{-5} \\ \alpha_{p_{22}} & =1.7153 \times 10^{2} & \alpha_{v_{1}} & =1.8560 \times 10^{-1} \\ \alpha_{v_{2}} & =2.9650 \times 10^{-4} & \alpha_{p} & =4.8000 \times 10^{-4} \\ \alpha_{s} & =1.0000 \times 10^{-3} & \alpha_{m} & =1.0000 \times 10^{-3}\end{aligned}$

which are specific free volume contributions of the individual species. The values of the gel and glass model parameters for the MMA polymerization system are given in Table 2 .

Under the assumption of $F_{i} \ll F_{m}$ and because the total volume of the reacting mixture in the reactor remains constant during operation (see the specifications of the experimental system), the volumetric flow rate of the outlet stream $(F)$ is related to that of the monomer stream $\left(F_{m}\right)$ (Schmidt and Ray, 1981) by:

$$
F=F_{m}\left(1+\vec{\epsilon} x_{p}\right),
$$

where the conversion $x_{p}$ is given by:

$$
x_{p}=\frac{\mu_{1}}{\mu_{1}+M_{A} C_{m}} .
$$

The constant $\bar{\epsilon}$ is a mean value of the volume expansion factor $\epsilon$ over the operating temperature range. The other kinetic and physical parameters for the system of MMA, AIBN, and toluene are given in Table 3.

Table 3. Physical and Kinetic Parameters (Schmidt and Ray, 1981; Louie et al., 1985)

$$
\begin{aligned}
& k_{\ell}=Z_{\ell} \exp \left(-E_{\ell} / R T\right) \ell=f_{m}, i, t_{0}, P_{0} \\
& Z_{t_{0}}=9.8000 \times 10^{7} \mathrm{~m}^{3} \cdot \mathrm{kmol}^{-1} \cdot \mathrm{s}^{-1} \\
& E_{t_{0}}=2.9442 \times 10^{3} \mathrm{~kJ} \cdot \mathrm{kmol}^{-1} \\
& Z_{P_{0}}=4.9167 \times 10^{5} \mathrm{~m}^{3} \cdot \mathrm{kmol}^{-1} \cdot \mathrm{s}^{-1} \\
& E_{P_{0}}=1.8283 \times 10^{4} \mathrm{~kJ} \cdot \mathrm{kmol}^{-1} \\
& Z_{f_{m}}=4.6610 \times 10^{9} \mathrm{~m}^{3} \cdot \mathrm{kmol}^{-1} \cdot \mathrm{s}^{-1} \\
& E_{f_{m}}=7.4479 \times 10^{4} \mathrm{~kJ} \cdot \mathrm{kmol}^{-1} \\
& Z_{i}=1.0533 \times 10^{15} \mathrm{~s}^{-1} \\
& E_{i}=1.2877 \times 10^{5} \mathrm{~kJ} \cdot \mathrm{kmol}^{-1} \\
& T_{g_{p}}=3.872 \times 10^{2} \mathrm{~K} \\
& T_{g_{m}}=1.672 \times 10^{2} \mathrm{~K} \\
& T_{g_{\mathrm{s}}}=1.132 \times 10^{2} \mathrm{~K} \\
& c=1.815 \times 10^{0} \mathrm{~kJ} \cdot \mathrm{kg}^{-1} \cdot \mathrm{K}^{-1} \\
& f=5.800 \times 10^{-1} \\
& m=8.646 \times 10^{\circ} \mathrm{kg} \\
& R=8.345 \times 10^{0} \mathrm{~kJ} \cdot \mathrm{kmol}^{-1} \cdot \mathrm{K}^{-1} \\
& \bar{\rho}_{m}=8.857 \times 10^{2} \mathrm{~kg} \cdot \mathrm{m}^{-3} \\
& p_{s}=8.155 \times 10^{2} \mathrm{~kg} \cdot \mathrm{m}^{-3} \\
& \rho_{p}=1.166 \times 10^{3} \mathrm{~kg} \cdot \mathrm{m}^{-3} \\
& M_{m}=1.0012 \times 10^{2} \mathrm{~kg} \cdot \mathrm{kmol}^{-1} \\
& M_{s}=9.214 \times 10^{1} \mathrm{~kg} \cdot \mathrm{kmol}^{-1} \\
& M_{i}=1.6421 \times 10^{2} \mathrm{~kg} \cdot \mathrm{kmol}^{-1} \\
& -\Delta H_{P}=5.780 \times 10^{4} \mathrm{~kJ} \cdot \mathrm{kmol}^{-1}
\end{aligned}
$$


We make the assumptions: (a) perfect mixing; (b) constant reacting mixture heat capacity $c$; (c) constant overall heattransfer coefficient $U$; (d) constant reacting mixture density; and (e) rate of chain-transfer-to-solvent reactions are not significant compared to the rates of other reactions. Under these assumptions, mole balances on the monomer, initiator and solvent, a mass balance on the dead polymer, and an energy balance for the reactor give the set of ordinary different equations:

$$
\left\{\begin{array}{l}
\frac{d C_{m}}{d t}=R_{m}\left(C_{m}, C_{i}, C_{s}, \mu_{1}, T\right)+\frac{C_{m_{m s}}-\left(1+\bar{\epsilon} x_{p}\right) C_{m}}{\tau} \\
\frac{d C_{i}}{d t}=R_{i}\left(C_{i}, T\right)+\frac{F_{i} C_{i_{i s}}-F_{m}\left(1+\bar{\epsilon} x_{p}\right) C_{i}}{V} \\
\frac{d C_{s}}{d t}=\frac{C_{s_{m s}} F_{m}+C_{s_{i s}} F_{i}-F_{m}\left(1+\bar{\epsilon} x_{p}\right) C_{s}}{V} \\
\frac{d \mu_{1}}{d t}=-M_{m} R_{m}\left(C_{m}, C_{i}, C_{s}, \mu_{1}, T\right)-\frac{\left(1+\bar{\epsilon} x_{p}\right) \mu_{1}}{\tau} \\
\frac{d T}{d t}=\frac{R_{H}\left(C_{m}, C_{i}, C_{s}, \mu_{1}, T\right) V}{m_{o_{r}} c}+\alpha_{1}\left(T_{j}-T\right)+\frac{T_{m s}-\left(1+\bar{\epsilon} x_{p}\right) T}{\tau}
\end{array}\right.
$$

where

$$
\alpha_{1} \triangleq \frac{A U}{m_{o_{r}} c}
$$

Note that, for simplicity of the mathematical model that will be used in the controller synthesis, a mean value of the volume expansion factor $(\bar{\epsilon})$ was used and the reacting mixture density was assumed to be constant. However, in the densitytemperature-conversion correlation (Eq. 1), the volume expansion factor $(\epsilon)$ is a function of temperature. This temperature dependence of $\epsilon$ is needed to account for the effect of temperature on the density measurements in the calculation of conversion.

\section{Jacket dynamics}

The jacket dynamics is described by the same jacket model given in our batch experimental study (Soroush and Kravaris, 1992a);

$$
\begin{aligned}
\frac{d T_{j}}{d t}=\alpha_{2}\left(T-T_{j}\right)+\alpha_{3}\left(T_{\infty}-\right. & \left.T_{j}\right) \\
& +\alpha_{4}\left[\mathbf{P}-F_{c w} \rho_{w} c_{w}\left(T_{j}-T_{c w}\right)\right],
\end{aligned}
$$

where

$$
\alpha_{2} \triangleq \frac{U A}{c_{w} m_{o_{j}}}, \quad \alpha_{3} \triangleq \frac{U_{\infty} A_{\infty}}{c_{w} m_{o_{j}}}, \quad \alpha_{4} \triangleq \frac{1}{c_{w} m_{o_{j}}}
$$

The overall dynamic model of the reactor (combining Eqs. 4 and 5), in a compact form, is given by:

$$
\left\{\begin{array}{l}
\frac{d C_{m}}{d t}=f_{m}\left(C_{m}, C_{i}, C_{s}, \mu_{1}, T\right) \\
\frac{d C_{i}}{d t}=f_{i}\left(C_{m}, C_{i}, \mu_{1}, T\right)+\frac{C_{i_{s}}}{V} u_{2} \\
\frac{d C_{s}}{d t}=f_{s}\left(C_{m}, C_{s}, \mu_{1}\right)+\frac{C_{s_{i s}}}{V} u_{2} \\
\frac{d \mu_{1}}{d t}=f_{\mu_{1}}\left(C_{m}, C_{i}, C_{s}, \mu_{1}, T\right) \\
\frac{d T}{d t}=f_{T}\left(C_{m}, C_{i}, C_{s}, \mu_{1}, T, T_{j}\right) \\
\frac{d T_{j}}{d t}=f_{T_{j}}\left(T, T_{j}\right)+\alpha_{4} u_{1}
\end{array}\right.
$$

where $u_{1}=\mathbf{P}-F_{c w} c_{w} \rho_{w}\left(T_{j}-T_{c w}\right), u_{2}=\mathbf{F}_{i}$ and

$$
f_{m}\left(C_{m}, C_{i}, C_{s}, \mu_{1}, T\right)=R_{m}\left(C_{m}, C_{i}, C_{s}, \mu_{1}, T\right)
$$

$$
+\frac{C_{m_{m s}}-\left(1+\bar{\epsilon} X_{p}\right) C_{m}}{\tau}
$$

$$
\begin{aligned}
f_{i}\left(C_{m}, C_{i}, \mu_{1}, T\right) & =R_{i}\left(C_{i}, \mathrm{~T}\right)-\frac{\left(1+\bar{\epsilon} x_{p}\right) C_{i}}{\tau} \\
f_{s}\left(C_{m}, C_{s}, \mu_{1}\right) & =\frac{C_{s_{m s}}-\left(1+\bar{\epsilon} X_{p}\right) C_{s}}{\tau} \\
f_{\mu_{l}}\left(C_{m}, C_{i}, C_{s}, \mu_{1}, T\right) & =-M_{m} R_{m}\left(C_{m}, C_{i}, C_{s}, \mu_{1}, T\right)-\frac{\left(1+\bar{\epsilon} X_{p}\right) \mu_{1}}{\tau} \\
f_{T}\left(C_{m}, C_{i}, C_{s}, \mu_{1}, T, T_{j}\right) & =\frac{R_{H}\left(C_{m}, C_{i}, C_{s}, \mu_{1}, T\right) V}{m_{o_{r}} c}+\alpha_{1}\left(T_{j}-T\right) \\
f_{T_{j}}\left(T, T_{j}\right) & =\alpha_{2}\left(T-T_{j}\right)+\alpha_{3}\left(T_{\infty}-T_{j}\right)
\end{aligned}
$$

In this study, the values of the four parameters, $\alpha_{1}, \alpha_{2}, \alpha_{3}$, and $\alpha_{4}$, are the same as the values of $\alpha_{1_{0}}, \alpha_{2_{0}}, \alpha_{3}$, and $\alpha_{4}$, respectively, in our batch experimental study (Soroush and Kravaris, 1992a).

\section{The GLC Method: a Review}

The GLC method is a framework for the synthesis of nonlinear control systems. Under a controller designed in this framework, the relations between the setpoints and the controlled outputs are described by linear ordinary differential equations. In other words, feedback is used to eliminate the process nonlinearities in an input/output sense. 
The first step in the GLC synthesis framework is the calculation of a static-state feedback, under which the input/ output behavior of the closed-loop system is exactly linear (see Kravaris and Soroush, 1990, for details). This means that in Figure 2a, the input/output behavior of the system inside the dotted line block ( $v-y$ system) is exactly linear. Once the closedloop $v-y$ system becomes linear, the controller synthesis problem reduces to the design of a linear controller with integral action. This linear controller is referred to as the external linear controller (see Figure 2a), which is needed for the controller robustness and the asymptotic rejection of process disturbances. The input/output stability and the degree of decoupling in the closed-loop system are determined by the choice of a set of adjustable parameters.

To implement the state feedback of the GLC, each process state variable should be measured, inferred, or estimated online. This practical consideration motivates the use of the general GLC structure in Figure 2b. In this structure, all the "useful" process measurements are used to infer information on the states. Those states, which cannot be measured or inferred, have to be estimated by using a reduced-order observer. A process measurement, which is not a controlled output and can be used to infer information on a state variable, will be called a secondary output. In Figure 2b, the vector of secondary outputs is represented by $\mathcal{Y}$.

A systematic and general approach for specifying the secondary outputs and synthesizing output feedback controllers within the GLC framework is given in the Appendix.

In this experimental study, in addition to the controlled outputs, the jacket temperature is measured and is a secondary output. A reduced-order observer is used for the estimation of the monomer, initiator, and solvent concentrations.

\section{Synthesis of the Control Law for the Experimental System}

To synthesize a nonlinear controller within the GLC framework for this experimental system, we follow the general approach in the Appendix.

The polymerization reactor model of Eq. 6 has six state variables ( $\left.x=\left[\begin{array}{llllll}C_{m} & C_{i} & C_{s} & \mu_{1} & T & T_{j}\end{array}\right]^{T}, n=6\right)$. The controlled outputs are the reactor temperature and conversion $(m=2)$ :

$$
y=\left[\begin{array}{c}
T \\
\frac{\mu_{1}}{M_{m} C_{m}+\mu_{1}}
\end{array}\right]
$$

and the manipulated inputs are the net heat input to the jacket $\left(u_{1}\right)$ and the inlet initiator flow rate $\left(F_{i}\right)$.

In addition to the controlled outputs ( $T$ and $x_{p}$ ), we can measure only the jacket temperature, which is one of the state variables and satisfies the condition of Eq. A2, that is, the $3 \times 6$ matrix,

$$
\left[\begin{array}{l}
\frac{\partial T_{j}}{\partial x} \\
\frac{\partial T}{\partial x} \\
\frac{\partial x_{p}}{\partial x}
\end{array}\right]
$$

has three linearly independent rows. Therefore, the jacket temperature is a secondary output $\left(\mathcal{Y}=T_{j}, s=1\right)$. Now we are in the position to proceed through Steps I to V (see the Appendix) and synthesize the controller of Eq. A15 for this experimental system:

(I) In this case, without rearranging the ordinary differential equations in Eq. 6, we see that the first three state variables, $C_{m}, C_{i}$ and $C_{s}$, satisfy the condition of Eq. A3:

$$
\operatorname{det}\left(\frac{\partial}{\partial x}\left[\begin{array}{c}
C_{m} \\
C_{i} \\
C_{s} \\
T_{j} \\
T \\
x_{p}
\end{array}\right]\right)=\operatorname{det}\left[\begin{array}{cccccc}
1 & 0 & 0 & 0 & 0 & 0 \\
0 & 1 & 0 & 0 & 0 & 0 \\
0 & 0 & 1 & 0 & 0 & 0 \\
0 & 0 & 0 & 0 & 0 & 1 \\
0 & 0 & 0 & 0 & 1 & 0 \\
* & 0 & 0 & * & 0 & 0
\end{array}\right] \neq 0
$$

where $*$ represents a nonzero term. Therefore, the model of Eq. 6 can equivalently be described in terms of the new state variables $C_{m}, C_{i}, C_{s}, T_{j}, T$, and $x_{p}$.

(II) Calculating the process model in terms of the new state variables:

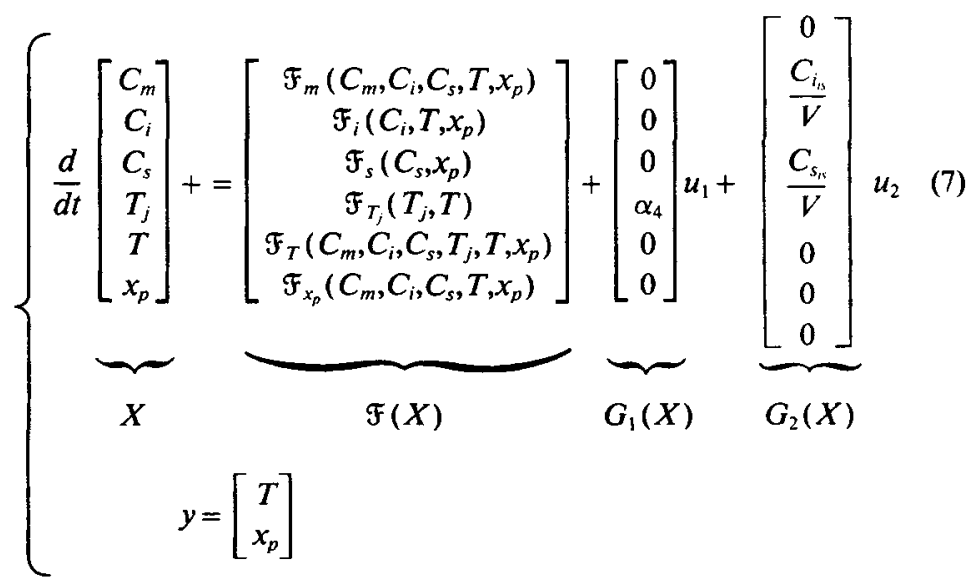




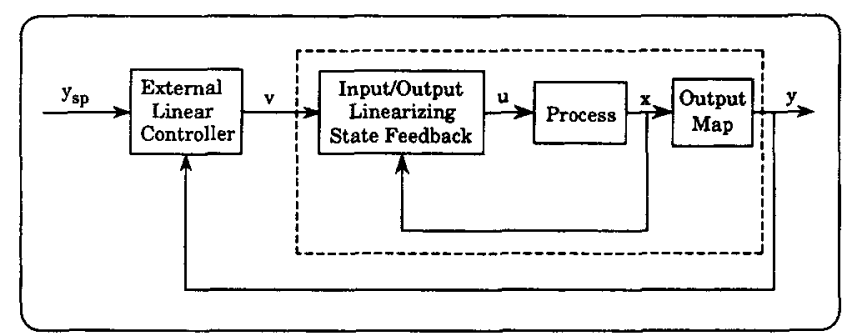

Figure 2a. GLC structure (full state information).

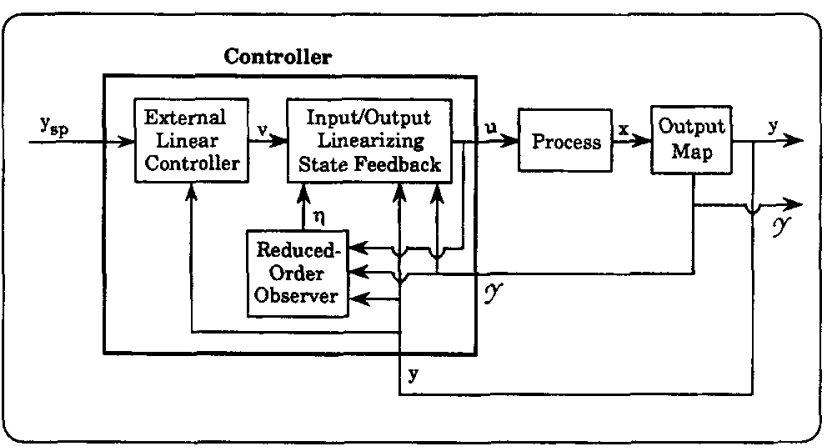

Figure 2b. GLC structure (incomplete state information).

where

$$
\begin{gathered}
\mathcal{F}_{m}\left(C_{m}, C_{i}, C_{s}, T, x_{p}\right)=f_{m}\left(C_{m}, C_{i}, C_{s}, \frac{M_{m} C_{m} x_{p}}{1-x_{p}}, T\right) \\
\mathcal{F}_{i}\left(C_{i}, T, x_{p}\right)=f_{i}\left(C_{m}, C_{i}, \frac{M_{m} C_{m} x_{p}}{1-x_{p}}, T\right) \\
\mathcal{F}_{s}\left(C_{s}, x_{p}\right)=f_{s}\left(C_{m}, C_{s}, \frac{M_{m} C_{m} x_{p}}{1-x_{p}}\right) \\
\mathcal{F}_{T_{j}}\left(T_{j}, T\right)=f_{T_{j}}\left(T, T_{j}\right) \\
\mathcal{F}_{T}\left(C_{m}, C_{i}, C_{s}, T_{j}, T, x_{p}\right)=f_{T}\left(C_{m}, C_{i}, C_{s}, \frac{M_{m} C_{m} x_{p}}{1-x_{p}}, T, T_{j}\right) \\
\mathcal{F}_{x_{p}}\left(C_{m}, C_{i}, C_{s}, T, x_{p}\right)=\left(1-x_{p}\right) \xi_{0}\left(C_{m}, C_{i}, C_{s}, x_{p}, T\right) \\
\quad \times\left[k_{P}\left(C_{m}, C_{s}, x_{p}, T\right)+k_{f_{m}}(T)\right]
\end{gathered}
$$

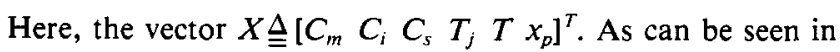
the above transformed process model (Eq. 7), the last three state variables are measured, whereas the first three state variables, $C_{m}, C_{i}$ and $C_{s}$, are not. The unmeasurable state variables, $C_{m}, C_{i}$ and $C_{s}$, can be reconstructed from on-line simulation of the first three ordinary differential equations in Eq. 7 :

$$
\left\{\begin{array}{l}
\frac{d \hat{C}_{m}}{d t}=\mathscr{F}_{m}\left(\hat{C}_{m}, \hat{C}_{i}, \hat{C}_{s}, T, x_{p}\right) \\
\frac{d \hat{C}_{i}}{d t}=F_{i}\left(\hat{C}_{i}, T, x_{p}\right)+\frac{C_{i_{i s}}}{V} u_{2} \\
\frac{d \hat{C}_{s}}{d t}=F_{s}\left(\hat{C}_{s}, x_{p}\right)+\frac{C_{s_{i s}}}{V} u_{2}
\end{array}\right.
$$

where $\hat{C}_{m}, \hat{C}_{i}$, and $\hat{C}_{s}$ are the estimated concentrations.

(III) For the system of Eq. 7, since

$$
\begin{aligned}
& G_{51}(X)=0 \quad G_{52}(X)=0, \quad G_{61}(X)=0, \quad G_{62}(X)=0 \\
& L_{G} \mathcal{F}_{T}(X)=\alpha_{1} \alpha_{4} \neq 0 \\
& L_{G_{2}} \mathcal{F}_{T}(X)=\frac{C_{i_{s}}}{V} \frac{\partial \mathcal{F}_{T}(X)}{\partial X_{2}}+\frac{C_{s_{i s}}}{V} \frac{\partial \mathcal{F}_{T}(X)}{\partial X_{3}} \not \equiv 0, \\
& L_{G_{1}} \mathfrak{F}_{x_{p}}(X)=0 \text {, } \\
& L_{G_{2}} \mathcal{F}_{x_{p}}(X)=\frac{C_{l_{i s}}}{V} \frac{\partial \mathcal{F}_{x_{p}}(X)}{\partial X_{2}}+\frac{C_{s_{s}}}{V} \frac{\partial \mathcal{F}_{x_{p}}(X)}{\partial X_{3}} \neq \equiv,
\end{aligned}
$$

and therefore, the relative orders $r_{1}=2$ and $r_{2}=2$, the characteristic matrix is:

$$
\mathcal{C}(X)=\left[\begin{array}{cc}
L_{G_{1}} \mathscr{F}_{r}(X) & L_{G_{2}} \mathcal{F}_{T}(X) \\
0 & L_{G_{2}} \mathcal{F}_{x_{p}}(X)
\end{array}\right]
$$

and the state feedback of Eq. A7 takes the form:

$$
u=\Psi\left(v, \hat{C}_{m}, \hat{C}_{i}, \hat{C}_{s}, T_{j}, T, x_{p}\right)
$$

where

$$
\Psi\left(v, \hat{C}_{m}, \hat{C}_{i}, \hat{C}_{s}, T_{j}, T, x_{p}\right)=\left[\mathcal{C}\left(\hat{C}_{m}, \hat{C}_{i}, \hat{C}_{s}, T, x_{p}\right)\right]^{-1}
$$

$$
\times\left[\begin{array}{c}
\frac{v_{1}-T-\sum_{\ell=1}^{2} \beta_{i p}^{1} L_{\mathcal{F}}^{\ell-1} \mathcal{F}_{T}\left(\hat{C}_{m}, \hat{C}_{i}, \hat{C}_{s}, T_{j}, T, x_{p}\right)}{\beta_{12}^{1}} \\
\frac{v_{2}-x_{p}-\sum_{\ell=1}^{2} \beta_{2 \ell}^{2} L_{\mathcal{F}}^{p-1} \mathfrak{F}_{x_{p}}\left(\hat{C}_{m}, \hat{C}_{i}, \hat{C}_{s}, T, x_{p}\right)}{\beta_{22}^{2}}
\end{array}\right]
$$

Here $\beta_{i j}^{\text {f's }}$ are scalar tunable parameter.

(IV) We use two SISO PI controllers as the external controller of the GLC:

$$
\begin{aligned}
& v_{1}=y_{s p_{1}}+K_{c_{1}}\left(e_{1}+\frac{1}{\tau_{l_{1}}} \int_{0}^{t} e_{1}(\tau) d \tau\right) \\
& v_{2}=y_{s p_{2}}+K_{c_{2}}\left(e_{2}+\frac{1}{\tau_{l_{2}}} \int_{0}^{\prime} e_{2}(\tau) d \tau\right)
\end{aligned}
$$

where $K_{c,}$ and $\tau_{i}$ are the gain and integral time constant of the $i$ th SISO PI controller. 
(V) Combining steps II, III and IV, we obtain the overall control system:

$$
\begin{cases}v_{1}=y_{s p_{1}}+K_{c_{1}}\left(e_{1}+\frac{1}{\tau_{I_{1}}} \int_{0}^{t} e_{1}(\tau) d \tau\right) & \\ v_{2}=y_{s p_{2}}+K_{c_{2}}\left(e_{2}+\frac{1}{\tau_{l_{2}}} \int_{0}^{t} e_{2}(\tau) d \tau\right) & \hat{C}_{m}(0)=C_{m}(0) \\ \frac{d \hat{C}_{m}}{d t}=\mathcal{F}_{m}\left(\hat{C}_{m}, \hat{C}_{i}, \hat{C}_{s}, T, x_{p}\right), & \hat{C}_{i}(0)=C_{i}(0) \\ \frac{d \hat{C}_{i}}{d t}=\mathcal{F}_{i}\left(\hat{C}_{i}, T, x_{p}\right)+\frac{C_{i_{i s}}}{V} F_{i}, & \hat{C}_{s}(0)=C_{s}(0) \\ \frac{d \hat{C}_{s}}{d t}=\mathcal{F}_{s}\left(\hat{C}_{s}, x_{p}\right)+\frac{C_{s_{i s}}}{V} F_{i}, & \\ u=\Psi\left(v, \hat{C}_{m}, \hat{C}_{i}, \hat{C}_{s}, T_{j}, T, x_{p}\right) & \end{cases}
$$

which induces the decoupled linear behavior

$\beta_{12}^{1} \frac{d^{3} y_{1}}{d t^{3}}+\beta_{11}^{\mathrm{\prime}} \frac{d^{2} y_{1}}{d t^{2}}+\left(K_{c_{1}}+1\right) \frac{d y_{1}}{d t}+\frac{K_{c_{1}}}{\tau_{J_{1}}} y_{1}=K_{c_{1}}\left(\frac{d y_{s p_{1}}}{d t}+\frac{1}{\tau_{l_{1}}} y_{s p_{1}}\right)$

$\beta_{22}^{2} \frac{d^{3} y_{2}}{d t^{3}}+\beta_{21}^{2} \frac{d^{2} y_{2}}{d t^{2}}+\left(K_{c_{2}}+1\right) \frac{d y_{2}}{d t}+\frac{K_{c_{2}}}{\tau_{I_{2}}} y_{2}=K_{c_{2}}\left(\frac{d y_{s p_{2}}}{d t}+\frac{1}{\tau_{I_{2}}} y_{s p_{2}}\right)$

to the closed-loop system.

\section{Practical Considerations}

Since water is used as the heating/cooling fluid in the jacket side, as a safety precaution, an upper limit is imposed on the jacket temperature $\left(T_{j} \leq 363.2 \mathrm{~K}\right)$ to prevent the formation of significant amount of water vapor in the heating/cooling circulation tubes. In the case that the jacket temperature exceeds 363.2 $\mathrm{K}$, the controller sets $P=0.6 \mathrm{~kJ} \cdot \mathrm{s}^{-1}$ to maintain the jacket temperature close to $363.2 \mathrm{~K}$ until this high jacket temperature is no longer required. This implies that whenever the state constraint is active, the upper constraint on the input $u_{1}$ is also active (the heater power is limited to $0.6 \mathrm{~kJ} \cdot \mathrm{s}^{-1}$ ). Therefore, in addition to manipulated input constraints $0 \leq F_{i} \leq F_{i_{\max }}, 0 \leq F_{\mathrm{cw}} \leq F_{\mathrm{cw}_{\max }}$ and $0 \leq \mathbf{P} \leq \mathbf{P}_{\max }$, there is an upper bound on the jacket temperature $\left(T_{j} \leq 363.2 \mathrm{~K}\right)$.

A key feature of this experiment is that during operation there are active state and input constraints. In particular, as we will see, the initiator flow rate remains at its upper or lower constraint for much longer time periods compared to other process variables that are bounded.

Once the manipulated inputs $u_{1}$ and $u_{2}$ are calculated from Eq. 10,

- The actual manipulated inputs $\mathbf{P}$ and $F_{c w}$ are calculated by using the same coordination rules as in Soroush and Kravaris (1992a), which indeed impose the manipulated input constraints on the controller action $u_{1}$, and
Table 4. Values of the Controller Tuning Parameters

$\begin{aligned} \beta_{11}^{1} & =2.1 \times 10^{3} \mathrm{~s} & \beta_{21}^{2} & =2.1 \times 10^{4} \mathrm{~s} \\ \beta_{12}^{1} & =2.0 \times 10^{5} \mathrm{~s}^{2} & \beta_{22}^{2} & =2.0 \times 10^{7} \mathrm{~s}^{2} \\ K_{c_{1}} & =1.0 \times 10^{1} & K_{c_{2}} & =1.0 \times 10^{1} \\ \tau_{1} & =2.1 \times 10^{3} \mathrm{~s} & \tau_{I_{2}} & =2.1 \times 10^{4} \mathrm{~s}\end{aligned}$

- The actual manipulated input $F_{i}$ is calculated from

$$
F_{i}=\left\{\begin{array}{lll}
0, & \text { if } & u_{2}<0 \\
u_{2}, & \text { if } & 0 \leq u_{2}<F_{i_{\max }} \\
F_{i_{\max }} & \text { if } & u_{2} \geq F_{i_{\max }}
\end{array}\right.
$$

which is the saturation function. To prevent reset windup in the two external PI controllers, whenever a manipulated input or state constraint is active, the integrator of a SISO PI controller is inactive.

The above approach for handling the constraints is intuitive (for a rigorous and "optimal" way of handling constraints, see Soroush, 1992). At the present time, the theoretical properties of the closed-loop system in the presence of active input or state constraints are unknown.

On the basis of the tuning guidelines given in Soroush and Kravaris (1992a), the controller-adjustable parameters are chosen. These controller parameters are given in Table 4.

\section{Implementation of the Control Law}

The following discrete-time version of the nonlinear controller is used in the computer code:

$$
u\left(t_{k}\right)=\Psi\left[v\left(t_{k}\right), \hat{C}_{m}\left(t_{k}\right), \hat{C}_{i}\left(t_{k}\right), \hat{C}_{s}\left(t_{k}\right), T_{j}\left(t_{k}\right), T\left(t_{k}\right), x_{p}\left(t_{k}\right)\right]
$$

where

- $\Psi$ is defined by Eq. 9

- $v\left(t_{k}\right)$ is calculated from

$$
v\left(t_{k}\right)=y_{s p}\left(t_{k}\right)+v^{\prime \prime}\left(t_{k}\right)
$$

$$
\begin{aligned}
v_{i}^{\prime \prime}\left(t_{k}\right)=v_{i}^{\prime \prime}\left(t_{k-1}\right)+ & K_{c_{i}}\left\{\left(\frac{\Delta t}{\tau_{I_{i}}}+1\right)\left[y_{s p_{i}}\left(t_{k}\right)-y_{i}\left(t_{k}\right)\right]\right. \\
& \left.-\left[y_{s p_{i}}\left(t_{k-1}\right)-y_{i}\left(t_{k-1}\right)\right]\right\}, \quad i=1,2
\end{aligned}
$$

where $\Delta t$ is the sampling period. Equation 14 is the velocity form of two SISO digital PI controllers.

- $\hat{C}_{m}\left(t_{k}\right), \hat{C}_{i}\left(t_{k}\right)$ and $\hat{C}_{s}\left(t_{k}\right)$ are calculated from numerical integration of the differential equations in Eq. 8 using the Runge-Kutta-Gill method.

Note that once the manipulated inputs $u_{1}\left(t_{k}\right)$ and $u_{2}\left(t_{k}\right)$ are calculated from Eq. 12, the corresponding values of the actual manipulated inputs $\mathbf{P}\left(t_{k}\right)$ and $F_{c w}\left(t_{k}\right)$ are calculated by using the coordination rules: 


$$
\mathbf{P}\left(t_{k}\right)= \begin{cases}u_{1}\left(t_{k}\right), & \text { if } 0 \leq u_{1}\left(t_{k}\right)<\mathbf{P}_{\max } \text { and } T_{j}\left(t_{k}\right) \leq 363.2 \mathrm{~K} \\ 0.60, & \text { if } 0 \leq u_{1}\left(t_{k}\right) \text { and } T_{j}\left(t_{k}\right)>363.2 \mathrm{~K} \\ \mathbf{P}_{\max }, & \text { if } u_{1}\left(t_{k}\right) \geq \mathbf{P}_{\max } \\ 0, & \text { if } u_{1}\left(t_{k}\right)<0\end{cases}
$$

2. Inferring the conversion $x_{p}\left(t_{k}\right)$ from $\rho\left(t_{k}\right)$ and $T_{d}\left(t_{k}\right)$ measurements (using Eq. 1).

3. Executing the PI controllers [calculating $v_{1}\left(t_{k}\right)$ and $v_{2}\left(t_{k}\right)$ from Eqs. 13 and 14 with $\tau_{l_{1}}$ and $\tau_{l_{2}}$ being calculated from Eqs. 17 and 18$]$.

$F_{c w}\left(t_{k}\right)= \begin{cases}\frac{-u_{1}\left(t_{k}\right)}{c_{w} \rho_{w}\left[T_{j}\left(t_{k}\right)-T_{c w}\right]}, & \text { if }-F_{c w_{\max }} c_{w} \rho_{w}\left[T_{j}\left(t_{k}\right)-T_{c w}\right] \leq u_{1}\left(t_{k}\right)<0 \\ F_{c w_{\max },} & \text { if } u_{1}\left(t_{k}\right)<-F_{c w_{\max }} c_{w} \rho_{w}\left[T_{j}\left(t_{k}\right)-T_{c w}\right] \\ 0, & \text { if } u_{1}\left(t_{k}\right) \geq 0\end{cases}$

and $F_{i}\left(t_{k}\right)$ is calculated by using the saturation function of Eq. 11. Furthermore, the integral action of the ith PI controller is "shut off" by setting $\tau_{l_{i}}=\infty$ (because of the use of velocity form PI controllers) when an input and/or a state constraint is active. In more precise terms, the antirest wind-up is achieved by setting $\tau_{I_{1}}$ and $\tau_{I_{2}}$ according to:

$$
\tau_{l_{1}}= \begin{cases}\infty, & \text { if } \mathbf{P}=\mathbf{P}_{\max } \text { or } F_{c w}=F_{c w_{\max }} \text { or } T_{j}>363.2 \mathrm{~K} \\ \beta_{11}^{1}, & \text { if otherwise }\end{cases}
$$

and

$$
\tau_{I_{2}}=\left\{\begin{array}{lll}
\infty, & \text { if } F_{i}=0 \text { or } F_{i}=F_{i_{\max }} \\
\beta_{21}^{2}, & \text { if } & \text { otherwise }
\end{array}\right.
$$

Figure 3 shows different blocks of the controller and process and their interconnections. The computer code of the controller includes the following steps in the order of their execution:

1 . Executing the sampling task at time $t_{k}$ (sampling period $\Delta t=5 \mathrm{~s})$. The jacket temperature $T_{j}\left(t_{k}\right)$ is the arithmetic mean of the measurements $T_{j_{\text {in }}}\left(t_{k}\right)$ and $T_{j_{\text {vet }}}\left(t_{k}\right)$.

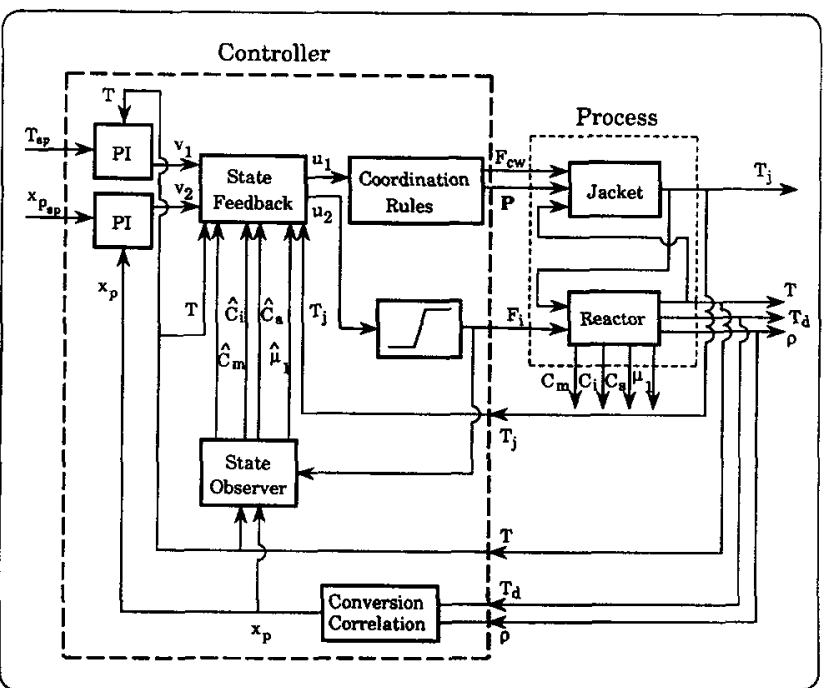

Figure 3. Block diagram of controller and process.
4. Executing the state estimator, that is, one-step-forward integration of the three differential equations:

$$
\left\{\begin{array}{l}
\frac{d \hat{C}_{m}}{d t}=f_{1}\left(\hat{C}_{m}, \hat{C}_{i}, \hat{C}_{s}, T, x_{p}\right), \\
\frac{d \hat{C}_{i}}{d t}=f_{2}\left(\hat{C}_{i}, T, x_{p}\right)+\frac{C_{i_{n}}}{\mathrm{~V}} \mathrm{~F}_{i}, \\
\frac{d \hat{C}_{s}}{d t}=f_{3}\left(\hat{C}_{s}, x_{p}\right)+\frac{C_{s_{n}}}{V} F_{i}
\end{array}\right.
$$

respectively, initialized at $\hat{C}_{m}\left(t_{k-1}\right), \hat{C}_{i}\left(t_{k-1}\right), \hat{C}_{s}\left(t_{k-1}\right)$ driven by the initiator flow rate $F_{i}\left(t_{k}\right)$ and the measurements of reactor temperature $T\left(t_{k}\right)$ and conversion $x_{p}\left(t_{k}\right)$, using the Runge-Kutta-Gill method (integration step size $=$ sampling period), to obtain $\hat{C}_{m}\left(t_{k}\right), \hat{C}_{i}\left(t_{k}\right)$, and $\hat{C}_{s}\left(t_{k}\right)$.

5. Executing the state feedback [calculating $u\left(t_{k}\right)$ from Eq. 12].

6. Executing the coordination rules and the saturation function [calculating $F_{i}\left(t_{k}\right), \mathbf{P}\left(t_{k}\right)$ and $F_{c w}\left(t_{k}\right)$ from Eqs. 11,15 and 16$]$.

7. Sending $\mathbf{P}\left(t_{k}\right), F_{c w}\left(t_{k}\right)$ and $F_{i}\left(t_{k}\right)$ signals to the heater, the control valve, and the initiator metering pump, respectively.

8. Returning to step 1.

The sequence of the above tasks is also shown in Figure 4, which is the flow diagram of the computer program.

By using the specific microcomputer, which is as fast as an IBM AT, the actual time (CPU time) needed for the execution of the controller system (SISO PI controllers, state observer, state feedback and coordination rules) is $0.2 \mathrm{~s}$, which is significantly less than the sampling period $(\Delta t=5 \mathrm{~s})$. This low value of CPU time on the specific microcomputer demonstrates the computational efficiency of the nonlinear control method. This computational efficiency becomes more significant when one considers the complexity of the polymerization model and the nonlinear-model-predictive nature of the controller (Soroush and Kravaris, 1992c). In the aforementioned work, it has been shown that when the GLC is applied to linear systems, the resulting controller is exactly a model algorithmic controller (MAC); the GLC is a nonlinear MAC. 


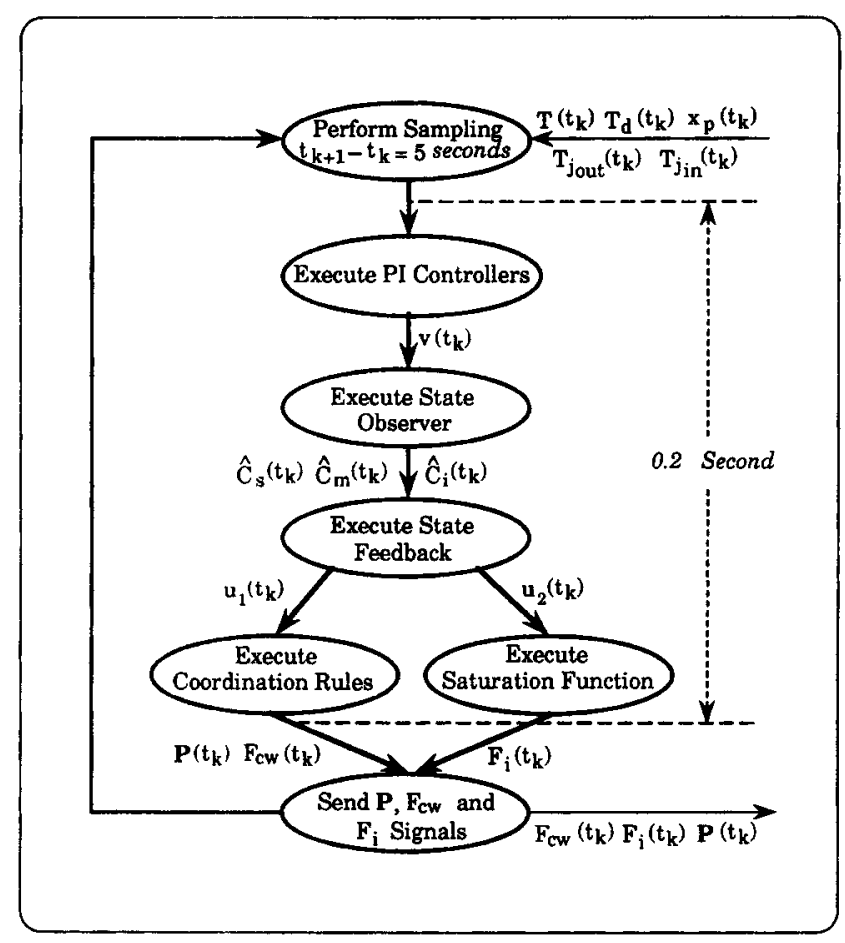

Figure 4. Flow diagram of computer program.

\section{Experimental Procedure}

After monomer purification (inhibitor removal, drying and vacuum distillation), the reactor is loaded with $1.2 \times 10^{-3} \mathrm{~m}^{3}$ of the monomer solution (composition by volume: $40 \%$ monomer and $60 \%$ toluene) at room temperature. The monomer feed bottles contain a solution of the same monomer-toluene composition. The concentration of the initiator solution in the initiator bottle is $0.2634 \mathrm{kmol} \cdot \mathrm{m}^{-3}$. The reason for using the above low monomer-to-solvent ratio and the conversion setpoint of 0.5 is the practical limitation imposed by the pressure drop across the density sensor (which exceeds $2.76 \times 10^{5}$ $\mathrm{kg} \cdot \mathrm{m}^{-1} \cdot \mathrm{s}^{-2}$ at the conversion of 0.5 ). Note that in developing the mathematical model, all important factors contributing to polymerization reactions in bulk were accounted for; therefore, if a higher monomer-to-solvent ratio is used, the controller and its execution time (CPU time) remain the same. In other words, a simpler model can describe the dynamics of the polymerization reactor under the above low monomer-to-solvent ratio. Despite using a very detailed model, the CPU time is $0.2 \mathrm{~s}$, which is significantly less than the sampling period.

Next, the monomer solution in the reactor and the monomer and initiator solutions in the feed bottles are purged of oxygen (a reaction inhibitor) by bubbling nitrogen through them for 1 hour. A blanket of nitrogen is also maintained over reacting liquids in the reactor and feed bottles during the operation.

The computer program is then initialized to perform reactor startup and then steady-state operation. Note that the reducedorder observer is initialized at the actual loading values of the concentrations $C_{m}, C_{i}$ and $C_{s}$. Our batch experimental study (Soroush and Kravaris, 1992a) showed that the controller is robust (insensitive) to errors in the initialization of the state observer.

The validity of the density-temperature-conversion corre-

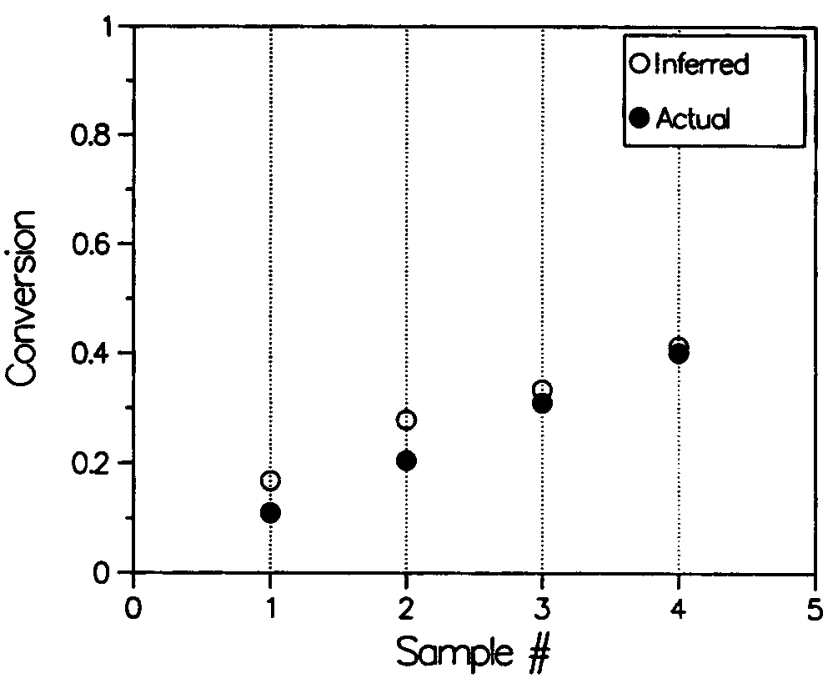

Figure 5. Comparison of actual and inferred values of conversion.

lation in inferring the conversion from the density and temperature measurements is checked by off-line measurements of the conversion using gravimetric methods (Collins et al., 1973), as shown in Figure 5. The accuracy of the densitytemperature-conversion correlation in inferring conversion increases as the steady-state conditions are approached. As mentioned earlier, the density measurements during the period of startup are overmeasured; the inferred values of the conversion are higher than actual values.

\section{Controller Performance}

The performance of the controller is investigated in terms of (a) performing a smooth and fast startup of the process and (b) tracking step changes in the setpoints.

Reactor Startup. Figure 6a depicts the profiles of the controlled outputs from the loading instant $(t=0)$ until the reactor reaches steady-state conditions. The loading conditions are given in Table 5. At $t=0$, only solvent and monomer are in the reactor $\left[x_{p}(0)=0\right]$ and the reactor is at room temperature. As this figure shows, the temperature response is faster than conversion response; it takes some time for the reactor to produce sufficient amount of polymer.

Figures $6 \mathrm{~b}, 6 \mathrm{c}$ and $6 \mathrm{~d}$ show the corresponding profiles of the inlet coolant flow rate $\left(F_{c w}\right)$, heater power $(\mathbf{P})$, initiator flow rate $\left(F_{i}\right)$, and jacket temperature $\left(T_{j}\right)$, respectively. As can be seen in Figures $6 b$ and $6 c$, the controller initially requests maximum heater power and inlet initiator flow rate (initially upper constraints on heater power and initiator flow rate are active); it wants to bring the controlled outputs to their setpoint values as soon as possible. The initiator flow rate after more than 1 hour of staying at its maximum value decreases to its lower limit and stays at zero for less than 1 hour, and finally it varies around its steady-state value. Figure $5 d$ shows that after a period of maximum heating, the jacket temperature exceeds $363.2 \mathrm{~K}$ (upper constraints on jacket temperature becomes active); therefore, as long as $T_{j}>363.2 \mathrm{~K}$, the controller sets the heater power $\mathbf{P}=0.60 \mathrm{~kJ} \cdot \mathrm{s}^{-1}$.

Step Changes in the Setpoints. Figure 7a depicts the pro- 


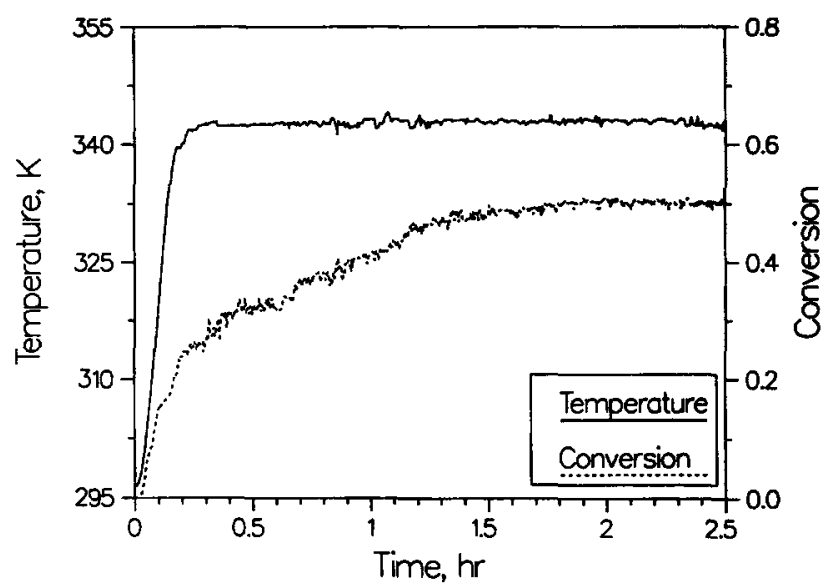

Figure 6a. Startup profiles of outputs.

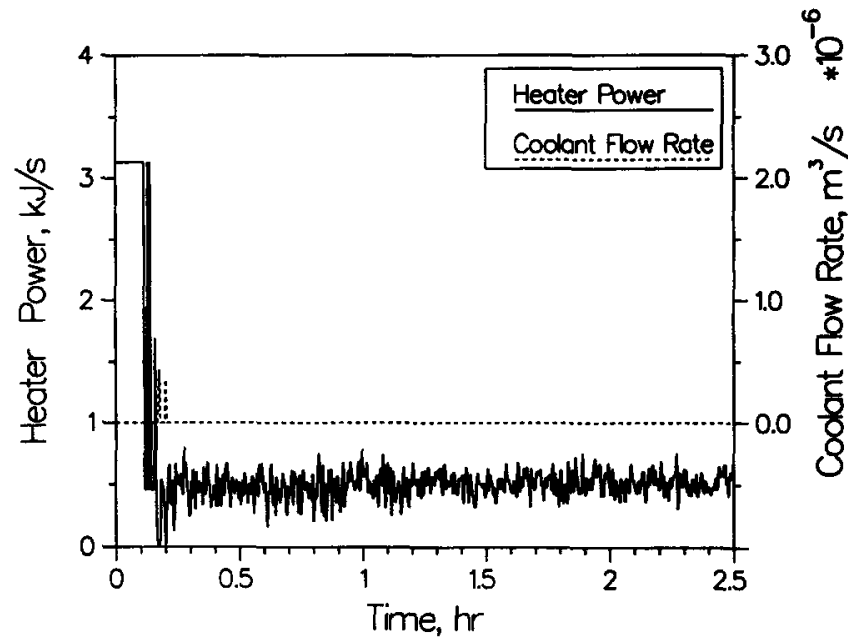

Figure 6b. Coolant flow rate and heater power corre. sponding to Figure 6 a.

files of the controlled outputs when there is a step change in the reactor temperature setpoint $T_{s p}$ from $343.2 \mathrm{~K}$ to $348.2 \mathrm{~K}$ at time $t=0.5 \mathrm{~h}$. The corresponding profiles of the inlet coolant flow rate $\left(F_{c w}\right)$, heater power $(\mathbf{P})$, initiator flow rate $\left(F_{i}\right)$ and jacket temperature $\left(T_{j}\right)$ are shown in Figures $6 \mathrm{~b}, 6 \mathrm{c}$ and $6 \mathrm{~d}$. Figure $7 \mathrm{~b}$ shows that the controller initially requests maximum

Table 5. Operating Conditions

$$
\begin{aligned}
C_{i}(0) & =0.000 \times 10^{0} \mathrm{kmol} \cdot \mathrm{m}^{-3} \\
C_{s}(0) & =5.881 \times 10^{0} \mathrm{kmol} \cdot \mathrm{m}^{-3} \\
C_{m}(0) & =3.984 \times 10^{0} \mathrm{kmol} \cdot \mathrm{m}^{-3} \\
\mu_{1}(0) & =0.000 \times 10^{0} \mathrm{kmol} \cdot \mathrm{m}^{-3} \\
T(0) & =2.952 \times 10^{2} \mathrm{~K} \\
T_{j}(0) & =2.952 \times 10^{2} \mathrm{~K} \\
C_{i_{\text {is }}} & =2.634 \times 10^{-1} \mathrm{kmol} \cdot \mathrm{m}^{-3} \\
C_{s_{i s}} & =8.986 \times 10^{0} \mathrm{kmol} \cdot \mathrm{m}^{-3} \\
C_{S_{m s}} & =5.881 \times 10^{0} \mathrm{kmol} \cdot \mathrm{m}^{-3} \\
C_{m_{m s}} & =3.984 \times 10^{0} \mathrm{kmol} \cdot \mathrm{m}^{-3} \\
T_{s p} & =3.432 \times 10^{2} \mathrm{~K} \\
x_{p_{\text {sp }}} & =5.000 \times 10^{-1}
\end{aligned}
$$

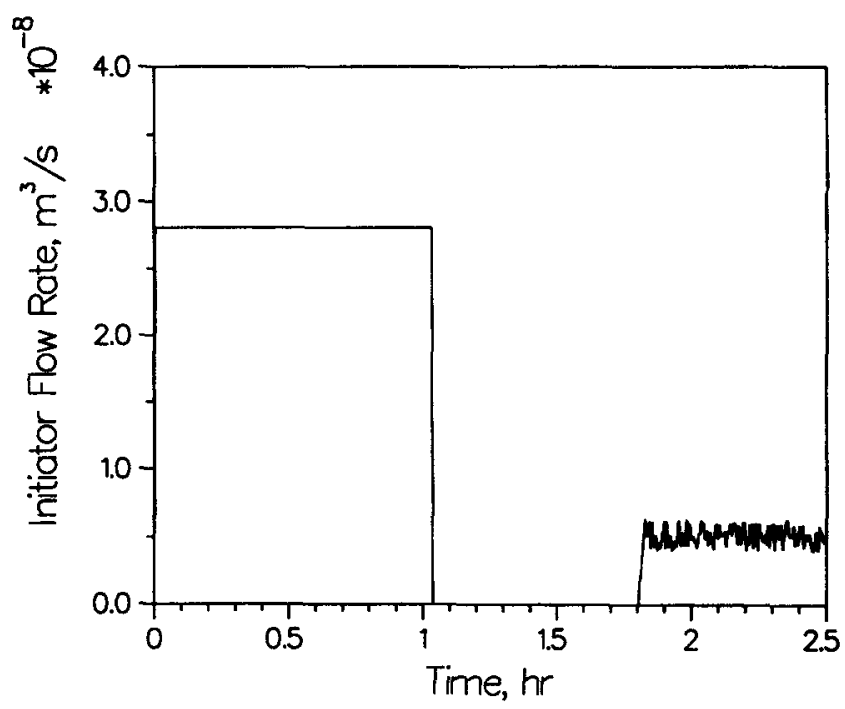

Figure 6c. Initiator stream flow rate corresponding to Figure 6a.

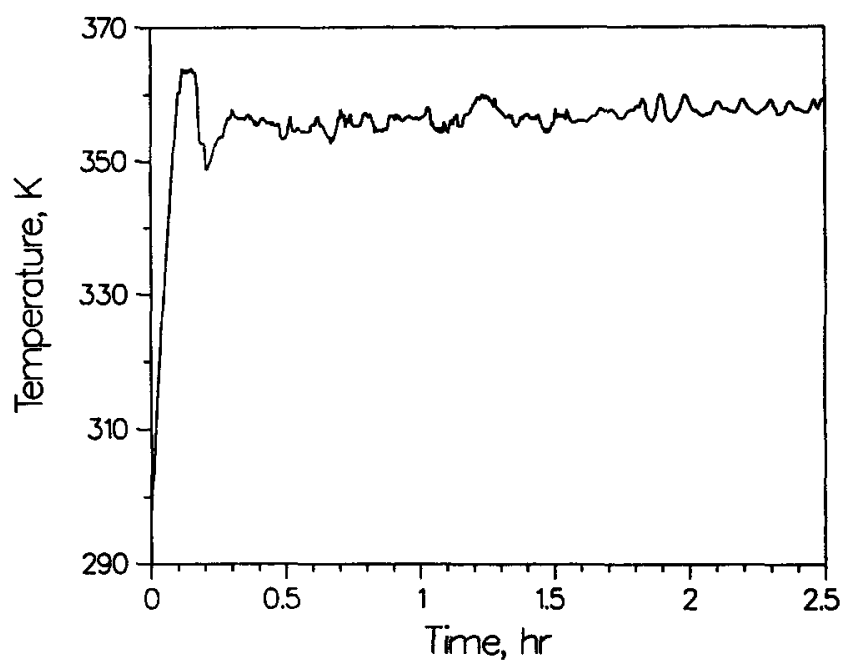

Figure 6d. Jacket temperature corresponding to Figure $6 a$.

heater power to bring the reactor temperature to its new setpoint value as soon as possible. Since the reactor has an interactive dynamics (a change in reactor temperature strongly affects the conversion), to compensate for this interaction (to eliminate the effect of the change in temperature on conversion) the controller stops adding initiator to the reactor ( $\operatorname{sets} F_{i}=0$ ). However, because the initiator flow rate calculated by the controller cannot be negative (initiator cannot be removed from the reactor), the controller cannot fully compensate for the effect of the temperature change on the conversion, and therefore, conversion increases. Conversion finally returns to its setpoint value after almost two hours. Figure $7 \mathrm{~d}$ shows that after a period of maximum heating, the jacket temperature exceeds $363.2 \mathrm{~K}$ (upper constraints on jacket temperature becomes active) and, as Figure $7 \mathrm{~b}$ shows, the heater power is set to $0.6 \mathrm{~kJ} \cdot \mathrm{s}^{-1}$ by the controller during this time-interval.

Figure $8 \mathrm{a}$ depicts the profiles of the controlled outputs when 


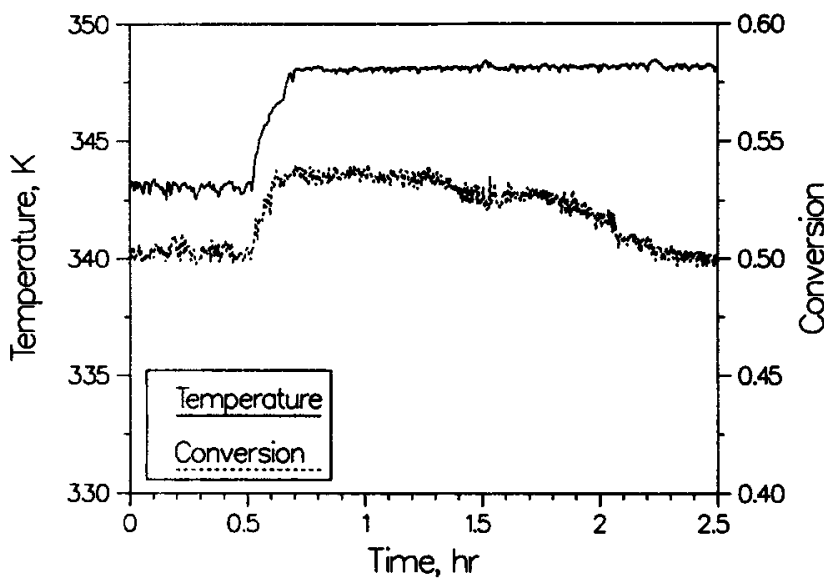

Figure 7a. Output variable responses to a step change in temperature setpoint at $t=0.5 \mathrm{~h}$.

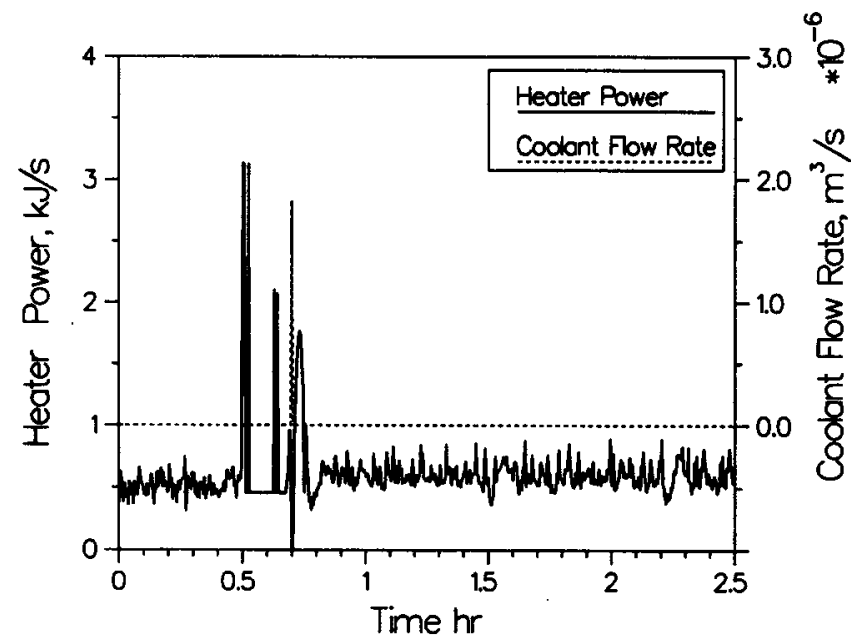

Figure $7 \mathrm{~b}$. Coolant flow rate and heater power corresponding to Figure 7 a.

there is a step change in the conversion setpoint $x_{p_{s p}}$ from 0.50 to 0.45 at time $t=0.5 \mathrm{~h}$. Like in the previous case, in which a step change was made in the temperature setpoint, here the conversion response is also very sluggish. In this case, the controller tries to bring conversion to its new setpoint value by only not adding initiator to the reactor. Ideally the controller wants to remove initiator from the reactor to decrease the rate of the chain initiation reactions and therefore decrease conversion. The lower limit on initiator flow rate is again responsible for the sluggishness of the conversion response. In contrast to the previous case, a step change in the conversion setpoint does not affect the other controlled output (reactor temperature), and the rate of heat input $u_{1}$ (calculated by the controller) remains almost unchanged [see the inlet coolant flow rate $\left(F_{c w}\right)$ and heater power $(\mathbf{P})$ profiles shown in Figure 8b]. This controller behavior can be explained as follows:

(a) Since the maximum flow rate of initiator is much smaller than the total inlet/outlet flow rate, a variation in the initiator flow rate does not have a significant effect on the rate of sensible heat to the reactor by the inlet streams.

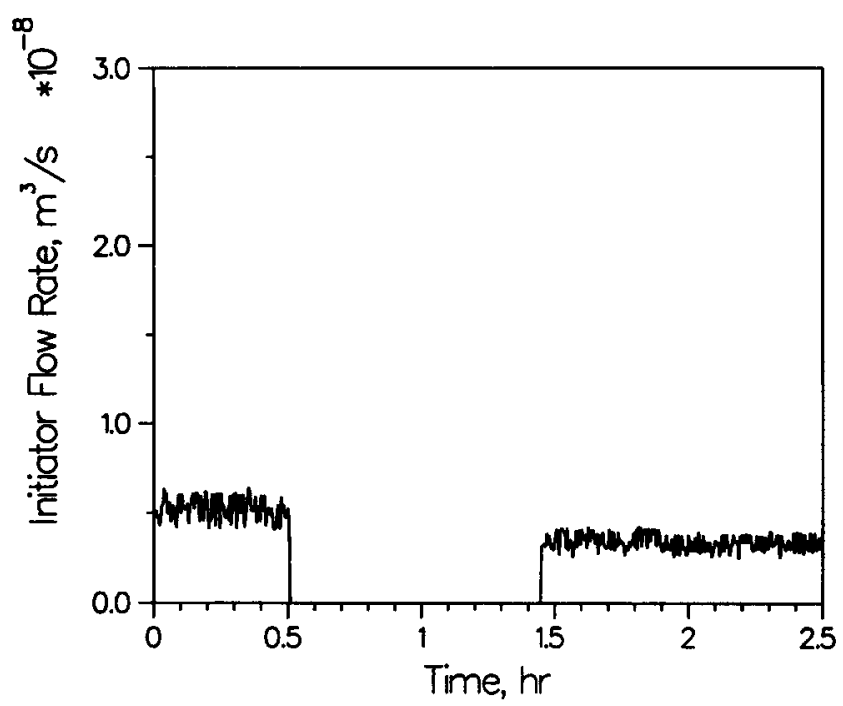

Figure 7c. Initiator stream flow rate corresponding to Figure 7a.

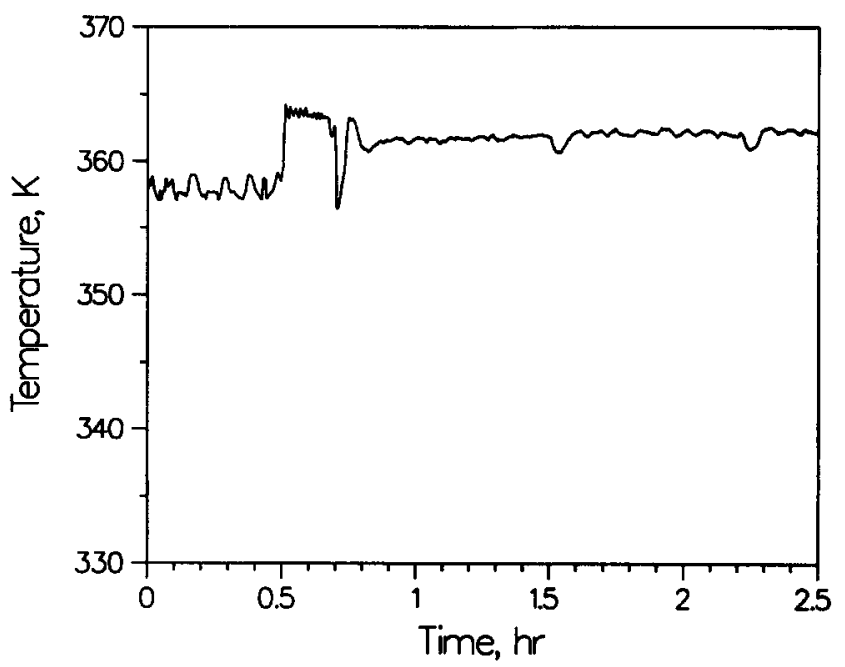

Figure 7d. Jacket temperature corresponding to Figure 7a.

(b) The overall rate of heat input to the reactor and heat production by the reactions should remain constant, since the reactor temperature is supposed to remain unchanged $\left(T_{s p}=348.2 \mathrm{~K}\right)$. For this case, the corresponding profiles of initiator flow rate $\left(F_{i}\right)$ and jacket temperature $\left(T_{j}\right)$ are depicted in Figures $8 \mathrm{c}$ and $8 \mathrm{~d}$.

(c) The temperature loop is much faster than the conversion loop; therefore, the temperature loop has adequate time to cancel the effect of decreased reaction rate (due to decrease in initiator concentration). Note that the high speed of the temperature loop is due to the high heat-transfer-surface-area-tovolume ratio of the reactor.

It is observed from Figures $7 \mathrm{a}$ and $8 \mathrm{a}$ that only one-way decoupling is achieved. The main reason for this is that the controller effort is limited by the lower constraint on the initiator flow rate (that is, initiator cannot be removed from the 


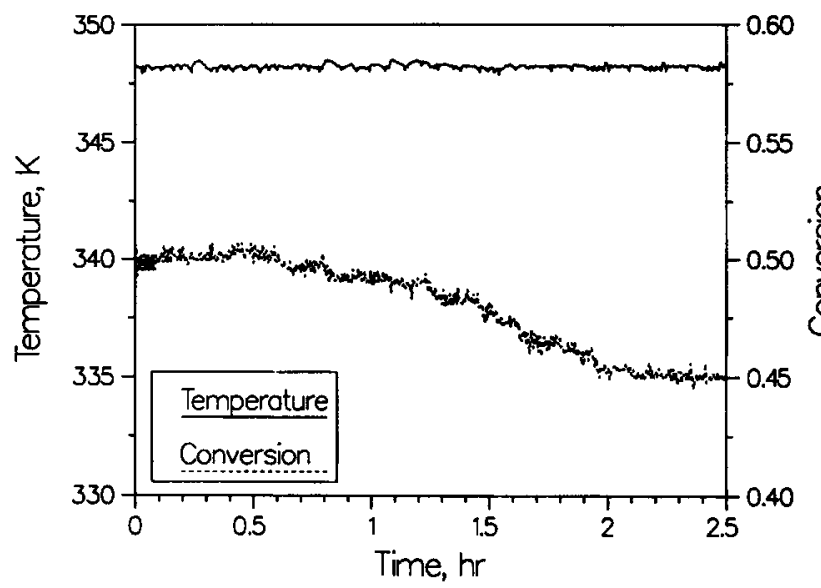

Figure 8a. Output variable responses to a step change in conversion setpoint at $t=0.5 \mathrm{~h}$.

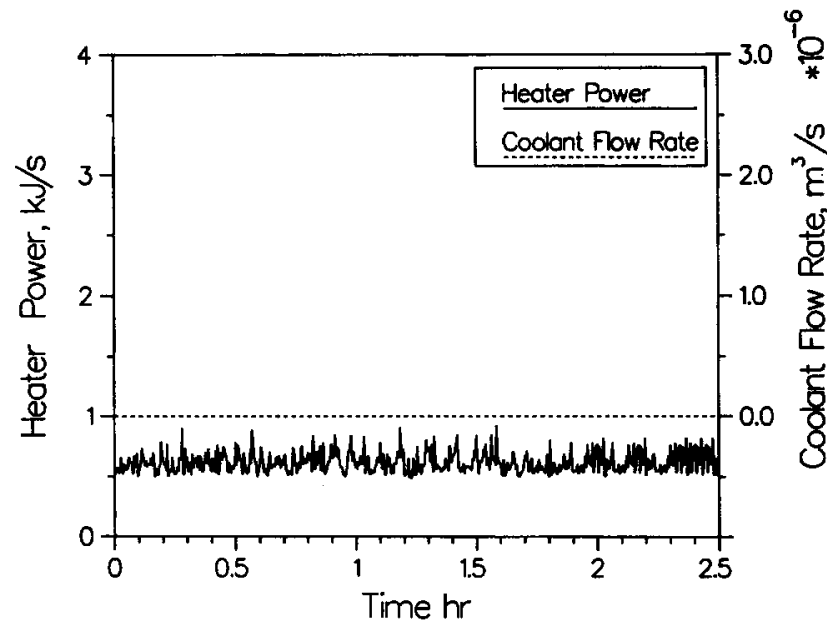

Figure $8 \mathrm{~b}$. Coolant flow rate and heater power corresponding to Figure 8 a.

system to return the conversion to its setpoint). A better conversion control can be achieved by using a "faster" initiator. However, this may lead to inhomogeneous polymerization (and hot spots). Note that the slow response of the conversion profile in Figures $7 \mathrm{a}$ and $8 \mathrm{a}$ is partially due to the presence of the lower constraint on the initiator flow rate.

\section{Conclusions}

A controller synthesized within the GLC framework was implemented experimentally to control conversion and temperature in a continuous stirred tank polymerization reactor. A general correlation for inferring conversion from density and temperature measurements was proposed. The performance of the controller in the presence of active input and state constraints was studied. In particular, it was shown that the tight constraints on the initiator flow rate have a strong deteriorating effect on the control quality. The experimental study showed that conversion control cannot be achieved effectively by manipulating the flow rate of the inlet initiator stream. This

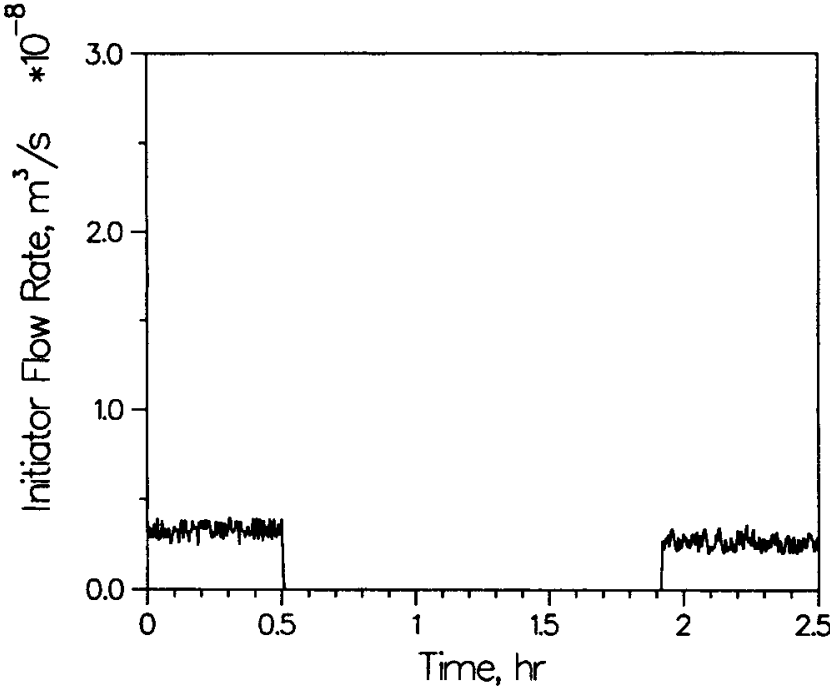

Figure 8c. Initiator stream flow rate corresponding to Figure 8a.

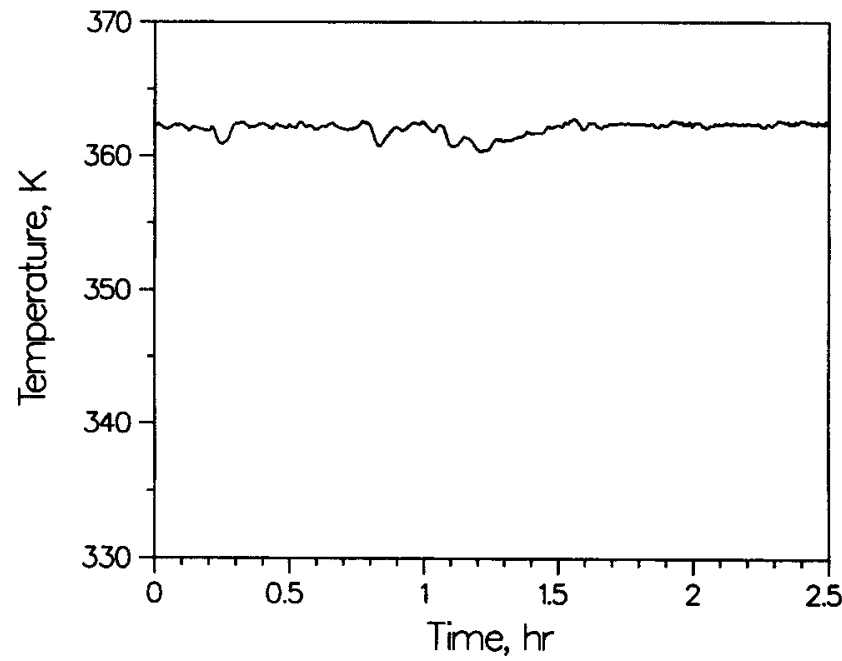

Figure 8d. Jacket temperature corresponding to Figure 8 .

work is the first experimental study in which a MIMO nonlinear model-based controller is applied to a polymerization reactor.

The presence of the active state and input constraints was treated intuitively, resulting in a satisfactory performance of the controller. A rigorous and theoretically supported way of handling input constraints is given in Soroush (1992).

The low value of CPU time on the specific microcomputer showed the considerable computational efficiency of the nonlinear control method. This low computational effort becomes more significant when one considers the complexity of the polymerization model, and the fact that the nonlinear controller is indeed a nonlinear-model-predictive controller (Soroush and Kravaris, 1992c).

\section{Acknowledgment}

Financial support from the National Science Foundation through the grant CTS-8912836 is gratefully acknowledged. 


\section{Notation}

$A, A_{\infty}=$ reactor-jacket and surrounding-jacket heat-transfer surface areas, $\mathrm{m}^{2}$

$c=$ heat capacity of reacting mixture, $\mathrm{kJ} \cdot \mathrm{kg}^{-1} \cdot \mathrm{K}^{-1}$

$c_{w}=$ heat capacity of water, $\mathrm{kJ} \cdot \mathrm{kg}^{-1} \cdot \mathrm{K}^{-1}$

$C_{1}=$ concentration of initiator, $\mathrm{kmol} \cdot \mathrm{m}^{-3}$

$C_{t_{s}}=$ concentration of initiator in inlet initiator stream, $\mathrm{kmol} \cdot \mathrm{m}^{-3}$

$C_{i}(0)=$ loading concentration of initiator, $\mathrm{kmol} \cdot \mathrm{m}^{-3}$

$C_{m}=$ concentration of monomer, $\mathrm{kmol} \cdot \mathrm{m}^{-3}$

$C_{m_{m},}=$ concentration of monomer in inlet monomer stream, $\mathrm{kmol} \cdot \mathrm{m}^{-3}$

$C_{m}(0)=$ loading concentration of monomer, $\mathrm{kmol} \cdot \mathrm{m}^{-3}$

$C_{s}=$ concentration of solvent, $\mathrm{kmol} \cdot \mathrm{m}^{-3}$

$C_{s_{s}}=$ concentration of solvent in inlet initiator stream, $\mathrm{kmol} \cdot \mathrm{m}^{-3}$

$C_{s_{\mathrm{m}}}=$ concentration of solvent in inlet monomer stream, $\mathrm{kmol} \cdot \mathrm{m}^{-3}$

$C_{s}(0)=$ loading concentration of solvent, $\mathrm{kmol} \cdot \mathrm{m}^{-3}$

$E_{i}, E_{P}=$ activation energies for initiation and propagation reactions, respectively, $\mathrm{kJ} \cdot \mathrm{kmol}^{-1}$

$E_{f_{m}}=$ activation energies for chain transfer to monomer reactions, $\mathrm{kJ} \cdot \mathbf{k m o l}^{-1}$

$E_{P_{0}}, E_{t_{0}}=$ activation energies for the reaction rate constants $k_{P_{0}}$ and $k_{t_{0}}$, respectively, $\mathrm{kJ} \cdot \mathrm{mol}^{-1}$

$f=$ initiator efficiency

$F_{c w}=$ inlet flow rate of cooling water, $\mathrm{m}^{3} \cdot \mathrm{s}^{-1}$

$F_{\left(w_{\max }\right.}=$ maximum inlet flow rate of cooling water, $\mathrm{m}^{3} \cdot \mathrm{s}^{-1}$

$F_{i}=$ flow rate of inlet initiator stream, $\mathrm{m}^{3} \cdot \mathrm{s}^{-1}$

$F_{i_{\max }}=$ maximum flow rate of initiator stream, $\mathrm{m}^{3} \cdot \mathrm{s}^{-1}$

$F_{m}=$ flow rate of inlet monomer stream, $\mathrm{m}^{3} \cdot \mathrm{s}^{-1}$

$g_{P}=$ ratio of apparent propagation rate constant to propagation rate constant at $x_{p}=0$

$g_{t}=$ ratio of apparent termination rate constant to termination rate constant at $x_{p}=0$

$I_{r}=\ell \times \ell$ identity matrix

$K_{c_{i}}=$ gain of the external controller of the $i$ th loop

$k_{f_{m}}, k_{P}=$ rate constants for chain transfer to monomer and propagation reactions, $\mathrm{m}^{3} \cdot \mathrm{kmol}^{-1} \cdot \mathrm{s}^{-1}$

$k_{i}=$ rate constant for initiation reaction, $\mathrm{s}^{-1}$

$k_{p}, k_{i}=$ overall propagation and termination rate constants, $\mathrm{m}^{3} \cdot \mathrm{kmol}^{-1} \cdot \mathrm{s}^{-1}$

$k_{P_{0}}, k_{t_{0}}=$ overall propagation and termination rate constants at zero conversion, $\mathrm{m}^{3} \cdot \mathrm{kmol}^{-1} \cdot \mathrm{s}^{-1}$

$m=$ mass of reacting mixture inside reactor, $\mathrm{kg}$

$m_{o_{1}}=$ overall effective mass of $\mathrm{H} / \mathrm{C}$ system, $\mathrm{kg}$

$m_{o_{r}}=$ overall effective mass of reacting mixture inside reactor, $\mathrm{kg}$

$M_{m}=$ molecular weight of monomer, $\mathrm{kg} \cdot \mathrm{kmol}^{-1}$

$M_{s}=$ molecular weight of solvent, $\mathrm{kg} \cdot \mathrm{kmol}^{-1}$

$\mathbf{P}=$ power input to heater, $\mathrm{kJ} \cdot \mathrm{s}^{-1}$

$\mathbf{P}_{\max }=$ maximum power of heater, $\mathrm{kJ} \cdot \mathrm{s}^{-1}$

$r_{i}=$ relative order of controlled output $y_{i}$

$R=$ universal gas constant, $\mathrm{kJ} \cdot \mathrm{kmol}^{-1} \cdot \mathrm{K}^{-1}$

$R_{H}=$ rate of heat production by propagation reactions, $\mathrm{kJ} \cdot \mathrm{m}^{-3} \cdot \mathrm{s}^{-1}$

$R_{m}, R_{i}=$ reaction rates for monomer and initiator, respectively, $\mathrm{kmol} \cdot \mathrm{m}^{-3} \cdot \mathrm{s}^{-1}$

$R_{x_{p}}=$ rate of increase in $x_{p}, \mathrm{~m}^{-3} \cdot \mathrm{s}^{-1}$

$R_{\mu_{1}}=$ rate of production of dead polymer, $\mathrm{kg} \cdot \mathrm{m}^{-3} \cdot \mathrm{s}^{-1}$

$t=$ time, $\mathrm{s}$

$T=$ reactor temperature, $\mathrm{K}$

$T_{c w}=$ temperature of inlet cooling water, $\mathrm{K}$

$T_{d}=$ temperature at which density is measured, $\mathrm{K}$

$T_{g m}=$ glass transition temperature of MMA, $\mathrm{K}$

$\underline{T}_{g p}=$ glass transition temperature of poly MMA, $\mathrm{K}$

$T_{g s}=$ glass transition temperature of solvent, $\mathrm{K}$

$T_{1}=$ temperature of inlet streams, $\mathrm{K}$

$T_{j}=$ jacket temperature, $\mathrm{K}$

$T_{j_{\mathrm{m}}}=$ temperature of jacket fluid before entering the jacket, $\mathrm{K}$

$T_{j \text { ou }}=$ temperature of jacket fluid after leaving the jacket, $\mathrm{K}$
$T_{m}=$ temperature of inlet monomer stream, $\mathrm{K}$

$T_{\infty}=$ room temperature, $\mathrm{K}$

$u=$ vector of manipulated inputs

$U, U_{\infty}=$ overall heat-transfer coefficients of reactor-jacket and jacket-surrounding, respectively, $\mathrm{kJ} \cdot \mathrm{m}^{-2} \cdot \mathrm{s}^{-1} \cdot \mathrm{K}^{-1}$

$v=$ vector of external inputs of linearized system

$V=$ volume of the reacting mixture, $\mathrm{m}^{3}$

$x=$ vector of state variables

$x_{p}=$ solvent-free mass-fraction of polymer in reactor

$y=$ vector of output variables

$Z_{i}=$ frequency factor for initiation reaction, $\mathrm{s}^{-1}$

$Z_{f_{m}}=$ frequency factor for chain transfer to monomer reactions, $\mathrm{m}^{3} \cdot \mathrm{kmol}^{-1} \cdot \mathrm{s}^{-1}$

$Z_{P_{0}}, Z_{t_{0}}=$ frequency factors for the reaction rate constants $k_{P_{0}}$ and $k_{t_{0}}$, respectively, $\mathrm{m}^{3} \cdot \mathrm{kmol}^{-1} \cdot \mathrm{s}^{-1}$

\section{Greek letters}

$\alpha_{i}=$ process and other constant parameters

$\beta_{j k}=m$-vector controller tunable parameters

$-\Delta H_{P}=$ heat of propagation reactions, $\mathrm{kJ} \cdot \mathrm{kmol}^{-1}$

$\Delta t=$ sampling period, $\mathrm{s}$

$\epsilon=$ polymerization volume expansion factor

$\vec{\epsilon}=$ mean value of volume expansion factor $\epsilon$ over operating temperature range

$\phi_{m_{m s}}=$ volume fraction of monomer in monomer stream $=C_{m_{m s}} M_{m} / \rho_{m}$

$\phi_{m}=$ volume fraction of monomer in reactor

$\phi_{p}=$ volume fraction of polymer in reactor

$\phi_{s}=$ volume fraction of solvent in reactor

$\mu_{1}=$ mass concentration of dead polymer chains, $\mathrm{kg} \cdot \mathrm{m}^{-3}$

$\rho=$ density of reacting mixture, $\mathrm{kg} \cdot \mathrm{m}^{-3}$

$\rho_{m}=$ density of monomer, $\mathrm{kg} \cdot \mathrm{m}^{-3}$

$\rho_{m s}=$ density of inlet monomer stream, $\mathrm{kg} \cdot \mathrm{m}^{-3}$

$\rho_{p}=$ density of polymer, $\mathrm{kg} \cdot \mathrm{m}^{-3}$

$\rho_{s}=$ density of solvent, $\mathrm{kg} \cdot \mathrm{m}^{-3}$

$\rho_{w}=$ density of water, $\mathrm{kg} \cdot \mathrm{m}^{-3}$

$\tau=$ CSTR residence time $=V / F_{m}, \mathrm{~s}$

$\tau_{I_{i}}=$ integral time constant of $i$ th PI controller

$\xi_{0}=$ molar concentration of live polymer chains, $\mathrm{kmol} \cdot \mathrm{m}^{-3}$

\section{Math symbols}

$$
\begin{aligned}
\triangleq & =\text { is defined } \\
\epsilon & =\text { belongs to } \\
\mathbf{R} & =\text { real line }
\end{aligned}
$$

$L_{f} h_{i}(x)=$ Lie derivative of the scalar field $h_{i}(x)$ with respect to the vector field $f(x)$

$$
=L_{f}^{\prime} h_{i}(x) \triangleq \sum_{\ell=1}^{n} \frac{\partial h_{i}(x)}{\partial x_{\ell}} f_{\ell}(x)
$$

$L_{f}^{\ell+1} h_{i}(x)=$ Lie derivative of scalar field $L_{f}^{\ell} h_{i}(x)$ with respect to vector field $f(x)$

$L_{g} L_{f}^{L} h_{i}(x)=$ Lie derivative of scalar field $L_{f}^{\mathcal{P}} h_{i}(x)$ with respect to vector field $g_{j}(x)$

\section{Literature Cited}

Amrehn, H., "Computer Control in Polymerization Industry," $A u$ tomatica, 13, 533 (1977).

Bejger, T. P., M. V. Tirrell, and G. Stephanopoulos, "Batch Copolymerization: Self Tuning Optimization and Control," AIChE Meeting, New Orleans (1981).

Chien, D. C. H., and A. Penlidis, "On-Line Sensors for Polymerization Reactors," JMS-Rev. Macromol. Chem. Phys., C30(1), 1 (1990).

Collins, E. A., J. Bares, and F. W. Billmeyer, Experiments in Polymer Science, Wiley, p. 333 (1973).

Elicabe, G. E., and G. R. Meira, "Estimation and Control in Polymerization Reactors: a Review," Poly. Eng. \& Sci., 28, 121 (1988). 
Inglis, M. P., W. R. Cluett, and A. Penlidis, "Long Range Predictive Control of a Polymerization Reactor," Can. J. of Chem. Eng., 69, 120 (1991).

Kravaris, C., and M. Soroush, "Synthesis of Multivariable Nonlinear Controllers by Input/Output Linearization," AIChE J., 36, 249 (1990).

Louie, B. M., G. M. Carratt, and D. S. Soong, "Modeling of the Radical Solution and Bulk Polymerization of Methyl Methacrylate," J. of Appl. Poly. Sci., 30, 3985 (1985).

McAuley, K. B., and J. F. MacGregor, "Nonlinear Multivariable Control of Product Properties in an Industrial Gas Phase Polyethylene Reactor," Proc. IFAC Symp. on Dynamics and Control of Chemical Reactors, Distillation Columns and Batch Processes, College Park, MD, 253 (1992).

MacGregor, J. F., "Control of Polymerization Reactors," Proc. of IFAC, 31 (1986).

Ponnuswamy, S. R., S. L. Shah, and C. A. Kiparissides, "On-Line Monitoring of Polymer Quality in a Batch Polymerization Reactor," J. of Appl. Polymer Sci., 32, 3239 (1986).

Ponnuswamy, S. R., S. L. Shah, and C. A. Kiparissides, "Computer Optimal Control of Batch Polymerization Reactors," Ind. Eng. Chem. Res., 26, 2229 (1987).

Ray, W. H., "Polymerization Reactor Control," IEEE Cont. Systs. Mag., 6(4), 3 (1986).

Ray, W. H., "Modeling and Control of Polymerization Reactors," Proc. IFAC Symp. on Dynamics and Control of Chemical Reactors, Distillation Columns and Batch Processes, College Park, MD, 161 (1992).

Schmidt, A. D., and W. H. Ray, "The Dynamic Behavior of Continuous Polymerization Reactors: I," Chem. Eng. Sci., 36, 1401 (1981).

Schork, F. J., and W. H. Ray, "On-Line Measurement of Surface Tension and Density with Application to Emulsion Polymerization," J. of Appl. Poly. Sci., 25, 407 (1983).

Singstad, P., H. Nordhus, K. Strand, M. Lien, L. B. Lyngmo, and O. Moen, "Multivariable Non-linear Control of Industrial LDPE Reactors," Proc. of ACC, 615 (1992).

Soroush, M., "Studies in Nonlinear Control and Optimal Design with Experimental Application to Polymerization Reactors," PhD Thesis, Univ. of Michigan, Ann Arbor (1992).

Soroush, M., and C. Kravaris, "Nonlinear Control of a Batch Polymerization Reactor: An Experimental Study," AIChE J., 38, 1429 (1992a).

Soroush, M., and C. Kravaris, "Multivariable Nonlinear Control of a Continuous Polymerization Reactor," Proc. of ACC, 607 (1992b),

Soroush, M., and C. Kravaris, "Discrete-Time Nonlinear Controller Synthesis by Input/Output Linearization," AIChE J., 38, 1923 (1992c).

Tirrell, M. V., R. Galvan, and R. L. Laurence, "Polymerization Reactors," Chemical Reaction and Reactor Engineering, J. J. Carberry and A. Varma, eds., Marcel Dekker, Basel-New York, 735 (1987).

Tirrell, M. V., and K. Gromley, "Composition Control of Batch Copolymerization Reactors," Chem. Eng. Sci., 36, 367 (1981).

Tzouanas, K. V., and S. L. Shah, "Adaptive Pole-Assignment Control of a Batch Polymerization Reactor," Chem. Eng. Sci., 5, 1183 (1989).

Weber, R., and C. B. Brosilow, "The Use of Secondary Measurements to Improve Control," AIChE J., 18, 614 (1972).

\section{Appendix: GLC Method for Processes with Sec. ondary Outputs}

A systematic approach is given for the synthesis of the GLCbased controllers for processes with secondary outputs (for details, see Soroush, 1992).

Consider nonlinear square (equal numbers of inputs and outputs) MIMO processes described by a state-space model of the form:

$$
\left\{\begin{array}{l}
\dot{x}(t)=f[x(t)]+\sum_{j=1}^{m} g_{j}[x(t)] u_{j}(t) \\
y(t)=h[x(t)]
\end{array}\right.
$$

where $x=\left[x_{1} \ldots x_{n}\right]^{T}, u=\left[u_{1} \ldots u_{m}\right]^{T}$ and $y=\left[y_{1} \ldots y_{m}\right]^{T}$ are the vectors of state variables, manipulated inputs and controlled outputs, respectively. Here, $g_{1}(x), \ldots, g_{m}(x)$, $h(x)=\left[h_{1}(x) \ldots h_{m}(x)\right]^{T}$ and $f(x)$ are analytic vector functions.

In general, in addition to the on-line measurements of the controlled outputs $y_{1}, \ldots, y_{m}$, there may be other on-line measurable process variables (denoted by $\mathcal{Y} \in \mathbb{R}^{5}$ ) that are algebraic functions of the state variables:

$$
\mathcal{Y}=\mathfrak{K}(x) .
$$

If these process variables (Y) satisfy the rank condition:

$$
\operatorname{rank}\left[\begin{array}{c}
\frac{\partial \mathcal{H}(x)}{\partial x} \\
\frac{\partial h(x)}{\partial x}
\end{array}\right]=s+m
$$

in a neighborhood of operating conditions, they can provide "useful" information on the process state variables. The condition of Eq. A2 means that the matrix:

$$
\left[\begin{array}{c}
\frac{\partial \mathcal{F C}(x)}{\partial x} \\
\frac{\partial h(x)}{\partial x}
\end{array}\right]
$$

should have $(s+m)$ linearly independent rows. If the condition of Eq. A2 is not satisfied, there must be at least a "redundant" or "unnecessary" process measurement; this can be resolved by dropping at least one of the extra measurements $\left(\mathcal{Y}_{1}, \ldots\right.$, $\mathcal{Y}_{s}$ ). Those extra measurable process variables, which satisfy the condition of Eq. A2, will be called the secondary outputs (to differentiate them from the controlled outputs $y_{1}, \ldots y_{m}$ ), and denoted by $Y \in \mathbb{R}^{s}$. The expression "secondary measurement" has also been used in cascade and inferential control (Weber and Brosilow, 1972) in other contexts. Note that the $m \times n$ matrix:

$$
\left[\frac{\partial h(x)}{\partial x}\right]
$$

will have $m$ linearly independent rows, if the control problem is defined well (system is output controllable).

After screening the extra process measurements and identifying the secondary outputs $(\mathcal{Y})$, a nonlinear controller within the GLC framework is synthesized as follows.

(I) Rearrange the ordinary differential equation in Eq. A1 so that the first $(n-s-m)$ state variables satisfy the condition: 


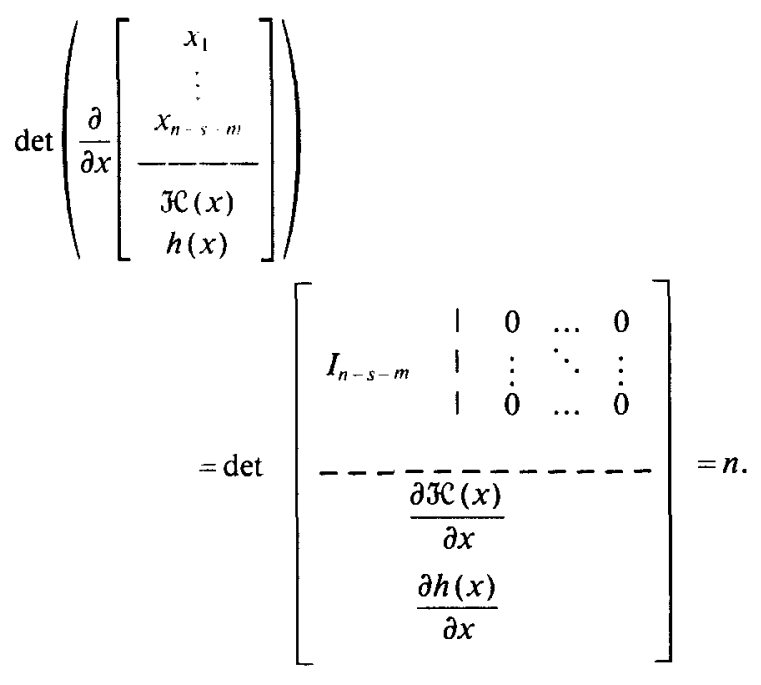

where

$$
\begin{aligned}
\mathcal{F}\left(x_{1}, \ldots, x_{n-m-s}, \mathcal{Y}, y\right) & =\left.\left(\frac{\partial \mathcal{J}(x)}{\partial x} f(x)\right)\right|_{x=J^{-1}\left(x_{i} \ldots \ldots, x_{n},-s, \mathcal{Y}, y\right)} \\
& =\left.\left(\frac{\partial J(x)}{\partial x} g_{j}(x)\right)\right|_{x=\mathcal{J}^{-1}\left(x_{1} \ldots, x_{n-m-s}, \mathcal{Y}, y\right)}
\end{aligned}
$$

As can be seen in the above transformed process model (Eq. A5), the last $(m+s)$ state variables are measured, whereas the first $(n-s-m)$ state variables $x_{1}, \ldots, x_{n-m-s}$ are not. The unmeasurable states can be reconstructed from on-line simulation of the first $(n-s-m)$ ordinary differential equations in Eq. A5:

$$
\left\{\begin{array}{c}
\dot{\hat{x}}=\mathfrak{F}_{1}\left(\hat{x}_{1}, \ldots, \hat{x}_{n-m-s}, \mathcal{Y}, y\right)+\sum_{j=1}^{m} G_{1 j}\left(\hat{x}_{1}, \ldots, \hat{x}_{n-m-s}, \mathcal{Y}, y\right) u_{j} \\
\vdots \\
\dot{\hat{x}}_{n-m-s}=\mathcal{F}_{n-m-s}\left(\hat{x}_{1}, \ldots, \hat{x}_{n-m-s}, \mathcal{Y}, y\right)+\sum_{j=1}^{m} G_{(n-m-s) j}\left(\hat{x}_{1}, \ldots, \hat{x}_{n-m-s}, \mathcal{Y}, y\right) u_{j}
\end{array}\right.
$$

in a neighborhood of operating conditions. Here for notational convenience, the vector of rearranged state variables is also denoted by $x$. Because of the condition of Eq. A2, this rearrangement of state variables is always possible. An importance of the condition of Eq. A3 is that any subset of unmeasurable state variables (for example, $x_{1}, \ldots, x_{n-s-m}$ ), that satisfies the rank condition of $\mathrm{Eq}$. $\mathrm{A} 3$, can be reconstructed by using a reduced-order observer. As a consequence of the condition of Eq. A3, the coordinate transformation:

$$
\left[\begin{array}{c}
x_{1} \\
\vdots \\
x_{n-s-m} \\
\mathcal{Y} \\
y
\end{array}\right]=\mathfrak{J}(x)=\left[\begin{array}{c}
x_{1} \\
\vdots \\
x_{n-s-m} \\
\mathfrak{H}(x) \\
h(x)
\end{array}\right]
$$

is invertible in a neighborhood of operating conditions. This means that the system of Eq. A1 can equivalently be described in terms of the variables $x_{1}, \ldots, x_{n-s-m}, \mathcal{Y}_{1}, \ldots, \mathfrak{Y}_{s}, y_{1}, \ldots$, $y_{m}$.

(II) Calculate the process model of Eq. A2 in the above new coordinate (in terms of the new state variables): if the subsystem of Eq. A6 is locally stable. Here, $\hat{x}_{1}, \ldots$, $\hat{x}_{n-s-m}$ are the estimated values of the unmeasurable states $x_{1}$, $\ldots, x_{n-s-m}$, respectively, and $G_{\ell j}$ is the th element of the vector $G_{j}$

(III) For the system of Eq. A6, calculate

(a) the relative orders $r_{1}, \ldots, r_{m}$, defined by:

$r_{i}=\left\{\begin{array}{cl}1, & \text { if there is an integer } j \text { such that } G_{(n-m+i) j} \not \equiv 0 \\ r_{j}^{*}>1 & \text { if for all } j G\left(_{n-m+i) j} \equiv 0\right.\end{array}\right.$

where $r_{i}^{*}$ is the smallest integer for which $\left[L_{G_{t}} L_{F}^{r_{i}^{*}-2} \mathcal{F}_{n-m+i} \ldots\right.$ $\left.L_{G_{m}} L_{\mathfrak{F}}^{r^{*}}{ }^{-2} F_{m-m+i}\right] \not \equiv[0 \ldots 0]$. (b) the characteristic matrix $\mathcal{C}\left(\hat{x}_{1}, \ldots, \hat{x}_{n-m-s}, \mathcal{Y}, y\right)$, which is an $m \times m$ matrix whose $i j$ th entry is given by:

$$
\begin{aligned}
\mathfrak{C}_{i j}\left(\hat{x}_{1}, \ldots, \hat{x}_{n-m-s}, \mathcal{Y}, y\right) & \begin{array}{ll}
G_{(n-m+i) j}\left(\hat{x}_{1}, \ldots, \hat{x}_{n-m-s}, \mathcal{Y}, y\right) & r_{i}=1 \\
L_{G_{j}} L_{\mathfrak{F}}^{r_{i}-2} \mathscr{F}_{n-m+i}\left(\hat{x}_{1}, \ldots, \hat{x}_{n-m-s}, \mathcal{Y}, y\right) & r_{i}>1
\end{array}
\end{aligned}
$$

$\left\{\begin{array}{c}\frac{d}{d t}\left[\begin{array}{c}x_{1} \\ \vdots \\ x_{n-m-s} \\ y \\ y\end{array}\right]=\mathscr{F}\left(x_{1}, \ldots, x_{n-m-s}, \mathcal{Y}, y\right)+\sum_{j=1}^{m} G_{j}\left(x_{1}, \ldots, x_{n-m-s}, \mathcal{Y}, y\right) u_{j} \\ y=y\end{array}\right.$ 
(c) an input/output linearizing state feedback of the form (provided that the characteristic matrix $\mathfrak{C}$ is nonsingular):

$$
\begin{aligned}
& u=\left\{\left[\beta_{1 r_{1}} \ldots \beta_{m r_{m}}\right] \mathbb{C}\left(\hat{x}_{1}, \ldots, \hat{x}_{n-m-s}, \mathcal{Y}, y\right)\right\}^{-1} \\
& \cdot\left\{v-y-\sum_{i=1}^{m} \sum_{\ell=1}^{r_{i}} \beta_{i \ell} L_{\mathcal{F}}^{\ell-1} \mathcal{F}_{n-m+i}\left(\hat{x}_{1}, \ldots, \hat{x}_{n-m-s}, \mathcal{Y}, y\right)\right\}
\end{aligned}
$$

where $\beta_{i \ell}=\left[\begin{array}{lll}\beta_{i \ell}^{1} & \ldots & \beta_{i \ell}^{m}\end{array}\right]^{T}$ are $m$-vector adjustable constant parameters with

$$
\operatorname{det}\left[\beta_{1 r_{1}} \ldots \beta_{m r_{m}}\right] \neq 0
$$

and $v \in \mathbb{R}^{m}$ is a reference input vector. The state feedback of Eq. A7 induces the linear input/output behavior:

$$
y+\sum_{i=1}^{m} \sum_{\ell=1}^{r_{i}} \beta_{i \ell} \frac{d^{\mathcal{P}} y_{i}}{d t^{\ell}}=v
$$

to the closed-loop system. In the above equations,

$$
v(t)=v_{b}(t)+\int_{0}^{t} \bar{G}_{e}(t-\tau) e(\tau) d \tau
$$

where $\bar{G}_{e}(t)$ is a matrix whose $i j$ th entry is the impulse response of the $i j$ th entry of the matrix transfer function $G_{e}(s)$, the error vector $e \triangleq y_{s p}-y$, and $v_{b}(t)$ is the external controller bias, given by (provided that the set-point profiles are smooth):

$$
v_{b}(t)=y_{s p}(t)+\sum_{i=1}^{m} \sum_{\ell=1}^{r_{i}} \beta_{i \ell} \frac{d^{\ell} y_{s p_{i}}(t)}{d t^{\ell}}
$$

In the case that a setpoint profile $y_{s p_{r}}(t)$ is piecewise constant, we set its time derivatives:

$$
\frac{d^{\ell} y_{s p_{i}}(t)}{d t^{\ell}} \equiv 0, \quad \ell=1,2, \ldots
$$

Note that the transfer function $G_{e}(s)$ should incorporate integral action; each element of the matrix transfer function $G_{e}(s)$ should have a pole at $s=0$.

(V) Combine the state observer (Eq. A6), the state feedback (Eq. A7), and the external controller (Eq. A10) and obtain the overall control system:

$$
\left\{\begin{aligned}
v & =v_{b}+\int_{0}^{t} \bar{G}_{e}(t-\tau) e(\tau) d \tau \\
\dot{\hat{x}}_{1}= & \mathcal{F}_{1}\left(\hat{x}_{1}, \ldots, \hat{x}_{n-m-s}, \mathcal{Y}, y\right)+\sum_{j=1}^{m} G_{1 j}\left(\hat{x}_{1}, \ldots, \hat{x}_{n-m-s}, \mathcal{Y}, y\right) u_{j} \\
\vdots & \\
\dot{\hat{x}}_{n-m-s}= & \mathcal{F}_{n-m-s}\left(\hat{x}_{1}, \ldots, \hat{x}_{n-m-s}, \mathcal{Y}, y\right)+\sum_{j=1}^{m} G_{(n-m-s) j}\left(\hat{x}_{1}, \ldots, \hat{x}_{n-m-s}, \mathcal{Y}, y\right) u_{j} \\
u= & \left\{\left[\beta_{1 r_{1}} \ldots \beta_{m r_{m}}\right] \mathbb{C}\left(\hat{x}_{1}, \ldots, \hat{x}_{n-m-s}, \mathcal{Y}, y\right)\right\}^{-1} \\
& \cdot\left\{v-y-\sum_{i=1}^{m} \sum_{\ell=1}^{r_{i}} \beta_{i \ell} L_{\mathfrak{F}}^{p-1} \mathcal{F}_{n-m+i}\left(\hat{x}_{1}, \ldots, \hat{x}_{n-m-s}, \mathcal{Y}, y\right)\right\}
\end{aligned}\right.
$$

$L_{\xi} h_{i}(x) \triangleq \sum_{\ell=1}^{n} \mathcal{F}_{\mathcal{P}}(x) \frac{\partial h_{i}(x)}{\partial x_{\ell}}, \quad L_{\mathcal{F}}^{\ell} h_{i}(x) \triangleq L_{\mathcal{F}}\left[L_{\mathcal{F}}^{\ell-1} h_{i}(x)\right]$

$$
\ell=1,2, \ldots
$$

$$
L_{\mathfrak{F}}^{0} h_{i}(x) \triangleq h_{i}(x), \quad L_{\mathfrak{F}} h_{i}(x) \triangleq L_{\mathfrak{F}}^{1} h_{i}(x)
$$

$$
L_{G_{j}} L_{\xi}^{\ell} h_{i}(x) \triangleq L_{G_{j}}\left[L_{\xi}^{\ell} h_{i}(x)\right], \quad \ell=0,1, \ldots
$$

(IV) Design and use a linear controller with integral action around the linear $(v-y)$ system, as the external controller of the GLC (see also Figure 2a). Let the matrix $G_{e}(s)$ be the transfer function of the external controller, therefore, which induces the following overall closed-loop input/output behavior (setpoint-output relation):

$$
\begin{aligned}
y+\sum_{i=1}^{m} \sum_{\ell=1}^{r_{i}} \beta_{i \ell} \frac{d^{\ell} y_{i}}{d t^{\ell}} & +\int_{0}^{t} \bar{G}_{e}(t-\tau) y(\tau) d \tau \\
& =y_{s p}+\sum_{i=1}^{m} \sum_{P=1}^{r_{i}} \beta_{i \ell} \frac{d^{\ell} y_{s p_{i}}}{d t^{\ell}}+\int_{0}^{t} \bar{G}_{e}(t-\tau) y_{s p}(\tau) d \tau
\end{aligned}
$$

if the setpoint profiles are smooth and the observer is initialized appropriately.

The control system of Eq. A11 represents a dynamic outputfeedback controller with integral action. The control structure is depicted in Figure $2 \mathrm{~b}$. In this figure, the vector of the estimated states $\left(\hat{x}_{1}, \ldots, \hat{x}_{n-m-s}\right)$ is represented by $\eta \in \mathbb{R}^{n-m-s}$.

Remark 1. The closed-loop system under the controller of Eq. A11 will be input/output stable, if the tunable parameters 
$\beta_{i f}$ and the external controller $G_{e}(s)$ are chosen such that the roots of:

$$
\begin{aligned}
\operatorname{det}\left\{I_{m}+\left[G_{e}(s)\right]^{-1}\right. & \\
& \left.\times\left\{I_{m}+\left[\left(\sum_{l=1}^{r_{1}} \beta_{1 \ell} s^{\ell}\right) \ldots\left(\sum_{l=1}^{r_{m}} \beta_{m l} s^{l}\right)\right]\right\}^{-1}\right\}=0
\end{aligned}
$$

lie inside the left half side of the complex plane. For an input/ output stable closed-loop behavior, the local internal stability (boundedness of all the states under the feedback) is guaranteed, if (a) the zero dynamics of the process and the system of Eq. Al are locally stable, and (b) the tunable parameters $\beta_{i p}$ are chosen so that the roots of:

$$
\operatorname{det}\left\{I_{m}+\left[\left(\sum_{\ell=1}^{r_{l}} \beta_{1 \ell} s^{\ell}\right) \ldots\left(\sum_{\ell=1}^{r_{m}} \beta_{m \ell} s^{\ell}\right)\right]\right\}=0
$$

lie inside the left hand side of the complex plane.

Remark 2. In the case that all the process state variables $(x)$ are measured on-line the control system of Eq. Al1 simplifies to:

$$
\left\{\begin{aligned}
& v=v_{b}+\int_{0}^{t} \bar{G}_{e}(t-\tau) e(\tau) d \tau \\
& u=\left\{\left[\beta_{1 r_{1}} \ldots \beta_{m r_{m}}\right] \mathbb{C}(x)\right\}^{-1} \\
& \times\left\{v-y-\sum_{i=1}^{m} \sum_{\ell=1}^{r_{i}} \beta_{i \ell} L_{\mathfrak{F}}^{\ell-1} \mathcal{F}_{n-m+i}(x)\right\}
\end{aligned}\right.
$$

with $\operatorname{det}\left[\beta_{1 r_{1}} \ldots \beta_{m r_{m}}\right] \neq 0$. The control structure of the above controller is depicted in Figure $2 a$.

Remark 3. In the case that input/output decoupling (in closed-loop, each setpoint $y_{s p_{i}}$ affects only the output $y_{i}$ ) is meaningful and desirable, it can be achieved by (a) selecting the tunable parameters $\beta_{i j}^{\ell}$ according to:

$$
\begin{aligned}
\beta_{i j}^{\ell}=0, \quad \ell \neq i, \quad \ell=1, \ldots, & m, \\
& j=1, \ldots, r_{i}, \quad i=1, \ldots, m
\end{aligned}
$$

with $\beta_{i r_{i}}^{i} \neq 0, i=1, \ldots, m$ (because of the condition of Eq. A8), and (b) using an external controller with diagonal transfer function ( $m$ SISO linear controllers in the external loop). In this case, the controller of Eq. All will significantly simplify. For instance, if the tuning parameters are chosen according to Eq. A14, $m$ SISO PI controllers are used in the external loop, and the setpoints are piecewise constant, the controller of Eq. Al1 will take the form:

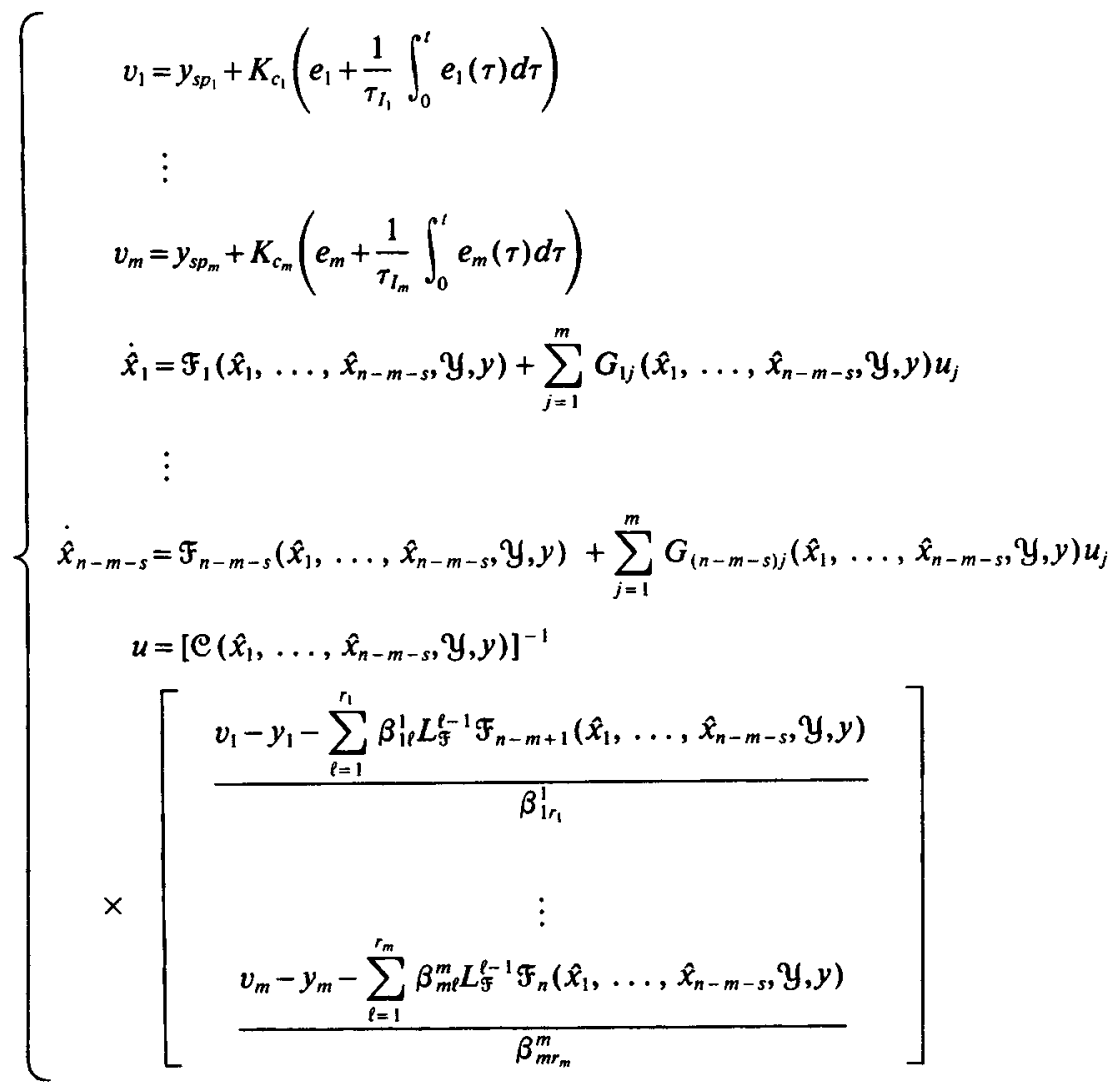

